\title{
Modelos para estimativa da radiação de onda longa atmosférica no cerrado Mato-Grossense
}

Models to the estimative of atmospheric long wave radiation in the cerrado of Mato Grosso

\section{Flair José Carrilho Sobrinho'; Soilce Beatriz de Paula Carrilho²; José de Souza Nogueira³; Osval- do Borges Pinto Junior ${ }^{4}$}

\author{
'Professor do Programa de Pós-Graduação em Física Ambiental, Linha de pesquisa: Análise e Modelagem de Processos Ecofisiológicos e \\ Análise Microclimática de Sistemas Urbanos, \\ 2Doutoranda do Programa de Pós-Graduação em Física Ambiental, Linha de pesquisa: Análise e Modelagem de Processos Ecofisiológicos, \\ ${ }^{3}$ Professor do Departamento de Física/ IF/ UFMT e Coordenador do Programa de Pós-Graduação em Física Ambiental,Professor da \\ Universidade de Cuiabá/ UNIC,
}

${ }^{4}$ Professor do Programa de Pós-Graduação em Física Ambiental, Linha de pesquisa: Análise Microclimática de Sistemas Urbanos,

\begin{abstract}
Resumo
Este trabalho teve por objetivo avaliar e parametrizar os modelos para a estimativa da radiação de onda longa atmosférica (Ld) no Cerrado Mato-grossense, considerando a cobertura do céu nas condições de céu-claro, parcialmente nublado e nublado. Levando-se em conta que medidas da Ld são raras, e que este parâmetro quase sempre é obtido de forma indireta através de vários modelos disponíveis na literatura, optou-se neste trabalho por analisar 107 modelos para a estimativa da Ld. Os dados utilizados neste trabalho foram obtidos de instrumentos instalados em uma torre microclimatológica de 19 metros na fazenda Miranda no município de Santo Antônio de Leverger - MT, entre os meses de junho e julho; e outubro e novembro de 2009 totalizando 5856 medidas. Das estimativas da Ld obtidas a partir destas formulações, aquelas com melhores desempenhos foram as que apresentaram como critérios, os menores erros estatísticos e os maiores índices e coeficientes como os de determinação (R2), de correlação de Pearson (r), de concordância de Wilmott (1982) e o de desempenho de Camargo \& Sentelhas (1997). Pode-se observar que, para as condições de céu claro, parcialmente nublado e nublado, os modelos de estimativa da irradiância de ondas longas, em sua formulação original, que apresentaram os melhores índices estatísticos e, portanto, aqueles que melhor se adaptaram ao Cerrado Mato-grossense, para o período estudado foram, respectivamente, Viswanadham \& Ramanadham (1970), Idso \& Jackson (1969) e Bignami et al. (1995) e após a parametrização os modelos de: Bárbaro et al. (2010); Aubinet (1994)) e Andreas \& Ackley (1982).
\end{abstract}

Palavras Chave: Índice de claridade; irradiância de ondas longas; equações empíricas; índices estatísticos, modelagem de ecossistemas.

\begin{abstract}
This study aimed to evaluate and parameterize models to estimating the atmospheric long wave radiation $(L d)$ in the Cerrado Mato-grossense, considering the sky coverage in terms of clear-sky, partly cloudy and overcast. Taking into account that measurements of $L d$ are rare, and that this parameter is usually obtained indirectly through various models available in the literature we decided in this study, analyze 107 models for the estimation of $L d$. The data used in this study were obtained of equipment installed in a micrometeorological tower 19 meters at Miranda farm in Santo Antônio de Leverger - MT, between the months of june and july, and october and november 2009 totalizing 5856 measures. Estimates of $L d$ obtained from these formulations, the best performing ones were the ones with the smallest statistical errors and the highest index and coefficients as of determination $\left(R^{2}\right)$, the Pearson correlation $(r)$, the Wilmott index of "agreement" (1982) $(d)$ and the Camargo \& Sentelhas index of "performance" (1997) (c). It can be observed that for conditions clear skies, partly cloudy and overcast, the models to estimate the long-wave irradiance, in its original formulation, which showed the best statistical indices and, therefore, those best adapted for the "Cerrado Mato-grossense" (Brazilian savanna in the Mato Grosso state), in the period studied were, respectively, Ramanadham \& Viswanadham (1970), Idso and Jackson (1969) and Bignami et al. (1995) and after parameterization of models: Bárbaro et al. (2010); Aubinet (1994) and Andreas \& Ackley (1982).
\end{abstract}

Keywords: Index of clarity, long-wave irradiance, empirical equations, statistical indices, ecosystem modeling. 


\section{INTRODUÇÃO}

O Cerrado é considerado a savana tropical mais diversa do mundo, sendo o segundo bioma em extensão no Brasil e representando cerca de $23 \%$ do território nacional, com uma área de 204,7 milhões de hectares.

A partir da década de 60 , foi iniciado um processo de antropização da região devido à mudança da capital federal para a região Centro-Oeste e a concessão de incentivos fiscais para a implantação de projetos agropecuários. Com isso, a ocupação da região dos Cerrados tem levado a problemas ambientais devido a não utilização de tecnologias adequadas e a grande extensão territorial das ocupações.

A sociedade, nos últimos anos, tem manifestado grande preocupação com os problemas ambientais, decorrentes da intensificação no uso de recursos e ambientes naturais associados ao processo produtivo com inapropriadas práticas de uso e manejo.

Em consequência, o ambiente físico (componentes climático, hidrológico e solo), biótico (fauna e flora) e socioeconômico vem sendo, constantemente, submetido a crescente pressão antropogênica, com efeitos que se traduzem em alterações de propriedades, estruturas, funções, inter-relações e sinergismos.

Neste sentido, conhecer a quantidade de radiação solar que chega à superfície terrestre é de grande importância para as diversas atividades humanas, principalmente aquelas referentes ao aproveitamento dos recursos naturais, visto que a radiação solar é a principal fonte de energia disponível a todos os processos físicos, químicos e biológicos que ocorrem no sistema terra-atmosfera, além de interferir em outros elementos meteorológicos como evaporação, evapotranspiração, temperatura, umidade relativa e outros.

A radiação solar ao penetrar na atmosfera sofre uma série de processos, provocando várias modificações, pois uma grande parte da radiação incidente é refletida pelas nuvens e difusamente espalhada pela atmosfera.

Quando ela interage com a atmosfera uma parte é absorvida pelas nuvens, aerossóis e moléculas que reemitem esta energia na forma de calor, sendo denominada radiação de ondas longas atmosféricas.

Assim como na Floresta Amazônica e no Pantanal, no Cerrado Mato-grossense praticamente não existem medidas regulares de radiação de onda longa da atmosfera, mesmo sendo uma variável importante no cálculo do balanço de radiação à superfície, pois representa a contribuição da atmosfera e engloba informações de nebulosidade e concentração de vapor d'água. Portanto, a utilização de equações analíticas e/ou empíricas para estimativa da irradiância de ondas longas tornou-se um modo alternativo largamente utilizado.

Neste contexto, o objetivo geral deste trabalho foi avaliar modelos analíticos e empíricos que consideram a cobertura do céu nas condições de céu-claro, parcialmente nublado e nublado para estimar a radiação solar de ondas longas proveniente da atmosfera em uma área representativa do Cerrado Mato-Grossense, chamado "Cerrado Sensu Stricto ".

\section{METODOLOGIA}

A pesquisa foi realizada na Fazenda

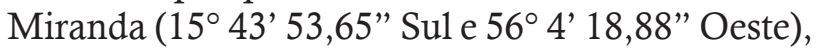
no município de Santo Antônio de Leverger situado na Baixada Cuiabana, Mato Grosso, Brasil, distante aproximadamente $15 \mathrm{~km}$ da cidade de Cuiabá e com altitude média de $157 \mathrm{~m}$.

Segundo a classificação de Köppen, o clima de Santo Antônio do Leverger é do tipo AW, também denominado Tropical semiúmido, possui quatro a cinco meses secos e duas estações bem definidas, uma seca (outono-inverno) e uma chuvosa (primavera-verão).

A formação vegetal predominante é o Cerrado (desde campo limpo até cerradão), apresentando floresta decídua na encosta dos morros e floresta de galeria ao longo dos rios, além de, em alguns trechos, vegetação típica de áreas alagadas.

O solo da área em que a pesquisa foi desenvolvida é caracterizado por ser pouco espesso, imperfeitamente drenado, concessionário e com superfície cascalhenta (Plintossolo Pétrico), podendo haver locais com solos Litólicos Distróficos (EMBRAPA, 1999).

$\mathrm{Na}$ área de estudo, numa torre em estrutura metálica de $19 \mathrm{~m}$ de altura com equipamentos de medições micrometeorológicas, permitiram-se a coleta e registros de dados, durante 24 horas por dia (Figura 2). Para realização deste trabalho, foram utilizados os dados micrometeorológicos coletados nos meses de junho, julho, outubro e novembro de 2009 por representar as duas estações definidas para o local de estudo: seca (junho e julho) e úmida (outubro e novembro).

As medidas de saldo de radiação $(R n)$ sobre a vegetação foram obtidas por meio de um saldo radiômetro NR LITE (Kipp e Zonen Delft, Inc., The Netherlands) instalado a $4 \mathrm{~m}$ de altura. A radiação global incidente $(R g i)$ e a radiação global refletida $(R g r)$ foram medidas por meio de pira- 


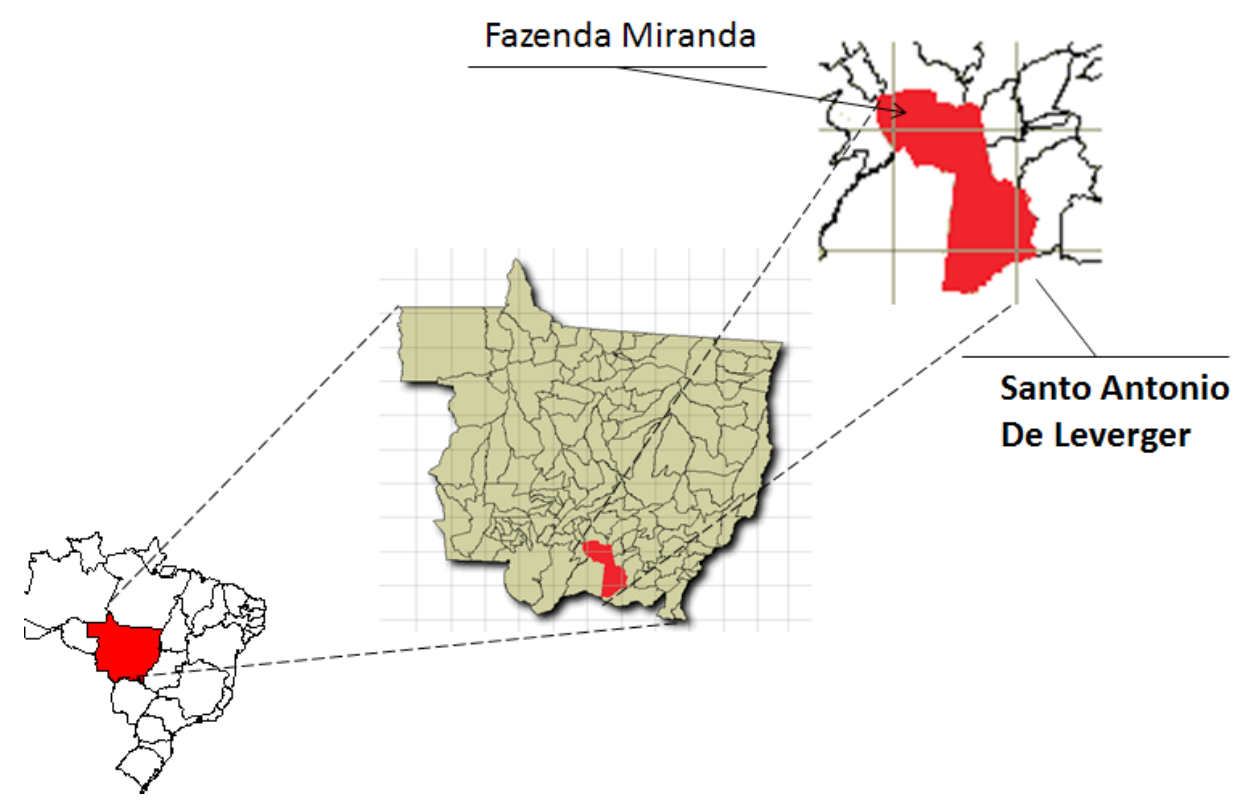

Figura1: Mapa do Brasil, Mato Grosso com a localização da área de estudo. Fonte: Adaptado de "http://www.ibge.gov.br/cidadesat/ acessado em 24/11/2011"
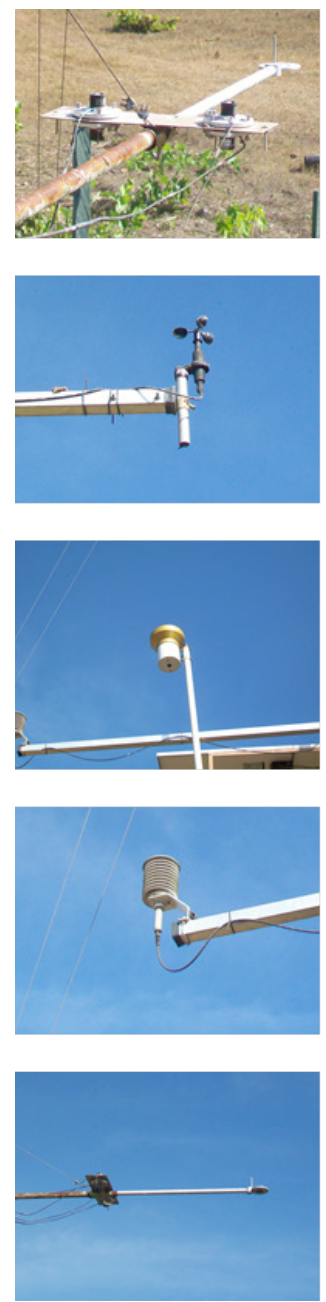
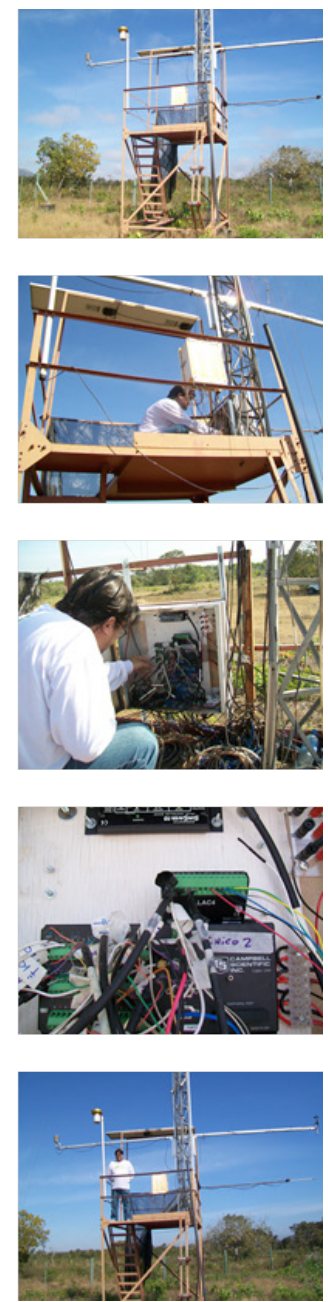

Figura 2: Torre micrometeorológica instalada na Fazenda Miranda, em Santo Antônio de Leverger, MT. Fonte: Acervo próprio. 
nômetros LI-200X-L (Campbell Scientific, Inc., USA) instalados a 4 metros de altura, um com a face voltada para cima ( $R g i)$ e o outro para baixo $(R g r)$, assim como a radiação fotossinteticamente ativa incidente $(P A R i)$ e a refletida $(P A R r)$, medidas através de sensores quantum LI-190SB-L (Campbell Scientific, Inc., USA), um com a face voltada para cima $(P A R i)$ e o outro para baixo $(P A R r)$. Os equipamentos da torre micrometeorológica foram alimentados com tensão de $12 \mathrm{~V}$ por uma bateria de $150 \mathrm{~A}$, carregada por meio de um painel solar de $65 \mathrm{~W}$ e com regulador de tensão.

Os dados micrometeorológicos foram coletados por meio de sensores de aquisição de dados, ligados a dois dataloggers CR 1000 (Campbell Scientific, Inc., USA), com intervalo de leitura de 30 segundos e registro das médias a cada 30 minutos.

Para aumentar o número de canais de entrada do registrador foi utilizada uma placa multiplexadora AM16/32a -ST-SW (Campbell Scientific, Inc., USA).

A cada 15 dias, os dados armazenados nos dataloggers eram transferidos para um disco rígido de um laptop. Verificações do funcionamento e o estado de conservação dos sensores também eram realizadas a cada visita no local.

Levando-se em conta que medidas de Ld são raras, este parâmetro quase sempre é obtido de forma indireta, através de vários modelos disponíveis na literatura, alguns dos quais têm validade somente para dias de céu claro. Para dias com nuvens devem ser efetuados ajustes para incluir os seus efeitos (BRUTSAERT, 1982). No presente trabalho Ld foi estimada por 107 modelos diferentes sendo 80 modelos para condições de céu claro $\left(\mathrm{Ld}_{\mathrm{cs}}\right)$ e 27 para condições de céu nublado $\left(\mathrm{Ld}_{\mathrm{cld}}\right)$ com os seus coeficientes originais, ou seja, foram preservados os valores originais propostos para os locais de estudo de cada modelo em condições de céu claro, parcialmente nublado e nublado segundo a classificação proposta por DALLACORT (2004).

Após as estimativas de Ld, os 107 modelos originais foram avaliados e classificados de acordo com os resultados obtidos dos índices estatísticos aplicados. Os modelos que obtiveram desempenho "Otimo" e "Muito bom" (Camargo e Sentelhas, 1997), ou seja, aqueles cujos valores estimados se aproximaram mais dos valores experimentais, tiveram os seus coeficientes ajustados nas condições do local de estudo e, novamente, avaliados e classificados buscando, assim, o modelo que melhor se adapta as condições no Cerrado Mato-grossense sob céu claro, parcialmente nublado e nublado.

Os 107 modelos utilizados para o cálculo da irradiância de ondas longas em condições de céu claro, parcialmente nublado e nublado foram categorizados de acordo com os seus parâmetros e se enquadraram em uma das oito categorias (Cat) na tabela 1.

Diversas estatísticas e técnicas citadas na literatura (Fox , 1980; Willmott et al., 1985; Yapo et al., 1998; Legates e Mccabe Jr., 1999; Tang et al., 2006; Duan et al., 2006) foram utilizadas para determinar o erro entre os dados simulados por um modelo $(P)$ e os dados observados $(O)$. Dentre elas destacam-se o coeficiente de correlação de Pearson $(r)$, o coeficiente de determinação $\left(R^{2}\right)$, o erro médio absoluto (EMA), o erro quadrático médio (EQM); o erro relativo médio $(E R M)$; o índice de concordância de Willmott (1982) - (d); o índice de confiança ou de desempenho de Camargo \& Sentelhas (1997) - (c); e a equação da reta de regressão dos dados simulados versus observados.

Além destas estatísticas para a avaliação dos modelos foram feitos, também, testes de normalidade e análise dos resíduos para um nível de significância de $5 \%$. O Teste de normalidade é utilizado para observar o comportamento da série de dados em estudo, ou seja, determina se os

Tabela 1: Categorias propostas de acordo com os parâmetros de cada modelo.

\begin{tabular}{|c|l|}
\hline Categorias & \multicolumn{1}{|c|}{ Parâmetros dos modelos em diferentes formas: linear, exponencial etc. } \\
\hline (CAT 1) & Tar - temperatura do ar próximo à superfície \\
\hline (CAT 2) & $e a$ - pressão de vapor d'água no ar próximo a superfície. \\
\hline (CAT 3) & Tar e $e a$ - temperatura do ar e pressão de vapor d'água no ar próximo a superfície. \\
\hline (CAT 4) & $T D P$ - temperatura do ponto de orvalho; Tar e/ou ea. \\
\hline (CAT 5) & $R H$ - umidade relativa do ar; $z$ - altitude local; Tar e/ou $e a$ \\
\hline (CAT 6) & $w$ - conteúdo d'água precipitável da atmosfera; Tar e/ou $e a$ \\
\hline (CAT 7) & $m$ - época do ano", mês do ano $(m=1,2,3, \ldots, 12) ;$ Tar e/ou $e a$ \\
\hline (CAT 8) & $q$ - umidade específica; $P 0$ - pressão atmosférica local; Tar e/ou $e a$ \\
\hline
\end{tabular}


dados seguem uma distribuição normal. Para isso existem diversos testes, sendo que para esse estudo utilizou-se os testes de Shapiro-Wilk, Anderson-Darling, Lilliefors, e Jarque-Bera para um nível de significância de $5 \%$.

\section{RESULTADOS E DISCUSSÕES}

Durante o período de estudo, entre os meses de junho e julho; e outubro e novembro de 2009, as condições meteorológicas em que as observações foram realizadas, caracterizam-se por apresentar variações semelhantes às observadas de acordo com as normais climatológicas da região (INMET 2009).

A radiação solar global incidente $(R g i)$ no período de estudo, variou de 3,63 $\mathrm{MJ}^{-2} \mathrm{~m}^{-2}$.dia ${ }^{-1}$ (valor mínimo) a $26,42 \mathrm{MJ} \cdot \mathrm{m}^{-2} \cdot \mathrm{dia}^{-1}$ (valor máximo) e valor médio de $16,49 \mathrm{MJ} \cdot \mathrm{m}^{-2}$.dia ${ }^{-1}$.

A tabela 2 ilustra as características meteorológicas do período estudado.

Os testes de normalidade Shapiro-Wilk, Anderson-Darling, Lilliefors, e Jarque-Bera, foram aplicados a todos os modelos e, para o nível de significância de $5 \%$, o p-valor obtido foi menor que $0,01 \%$, logo, como o p-valor calculado é menor que o nível de significância $\alpha=5 \%$, deve-se rejeitar a hipótese nula $\mathrm{H} 0$ em favor da hipótese alternativa $\mathrm{Ha}$, ou seja, todos os modelos analisados seguem uma distribuição normal. A tabela 3 mostra os resultados dos testes de normalidade para todos os modelos.

Tabela 2: Evolução da Cobertura do céu (\% dos dias estudados), da Temperatura média mensal $\left({ }^{\circ} \mathrm{C}\right)$, da $\mathrm{R}_{\mathrm{gi}}\left(\mathrm{MJ} \cdot \mathrm{m}^{-2}\right.$. mês $\left.{ }^{-1}\right)$ e da Precipitação mensal acumulada (mm) em cada mês do período de estudo

\begin{tabular}{|c|c|c|c|c|c|c|}
\hline Mês & $\begin{array}{c}\text { Céu } \\
\text { Claro }\end{array}$ & $\begin{array}{c}\text { Céu } \\
\text { Parcialmente } \\
\text { Nublado }\end{array}$ & $\begin{array}{c}\text { Céu } \\
\text { Nublado }\end{array}$ & $\begin{array}{c}\text { Temperatura } \\
\text { média mensal }\end{array}$ & $\begin{array}{c}\text { Radiação } \\
\text { solar } \\
\text { global } \\
\text { incidente }\end{array}$ & $\begin{array}{c}\text { Precipitação } \\
\text { mensal } \\
\text { acumulada }\end{array}$ \\
\hline Junho & $43 \%$ & $37 \%$ & $20 \%$ & 22,22 & 411,58 & 57,15 \\
\hline Julho & $39 \%$ & $51 \%$ & $10 \%$ & 23,50 & 484,47 & 12,99 \\
\hline Outubro & $7 \%$ & $87 \%$ & $6 \%$ & 27,83 & 572,44 & 96,52 \\
\hline Novembro & $3 \%$ & $83 \%$ & $14 \%$ & 27,64 & 543,49 & 183,20 \\
\hline
\end{tabular}

Tabela 3: Resultados dos testes de normalidade para $\alpha=5 \%: \mathrm{H} 0 \rightarrow$ A amostra segue uma distribuição Normal quando $\mathrm{p}$-valor $<\alpha ; \mathrm{Ha} \rightarrow \mathrm{A}$ amostra não segue uma distribuição Normal quando $\mathrm{p}$-valor $>\alpha$.

\begin{tabular}{|l|c|c|c|c|}
\hline \multicolumn{1}{|c|}{ Modelo } & Shapiro-Wilk & Anderson-Darling & Lilliefors & Jarque-Bera \\
\hline Angström (1918) & p-valor $<0,01 \%$ & p-valor $<0,01 \%$ & p-valor $<0,01 \%$ & p-valor $<0,01 \%$ \\
\hline Kimball (1918) & p-valor $<0,01 \%$ & p-valor $<0,01 \%$ & p-valor $<0,01 \%$ & p-valor $<0,01 \%$ \\
\hline Robitsch (1926) & p-valor $<0,01 \%$ & p-valor $<0,01 \%$ & $\mathrm{p}$-valor $<0,01 \%$ & $\mathrm{p}$-valor $<0,01 \%$ \\
\hline Dines \& Dines (1927) & $\mathrm{p}$-valor $<0,01 \%$ & $\mathrm{p}$-valor $<0,01 \%$ & $\mathrm{p}$-valor $<0,01 \%$ & $\mathrm{p}$-valor $<0,01 \%$ \\
\hline Asklef (1928) & $\mathrm{p}$-valor $<0,01 \%$ & $\mathrm{p}$-valor $<0,01 \%$ & $\mathrm{p}$-valor $<0,01 \%$ & $\mathrm{p}$-valor $<0,01 \%$ \\
\hline Boutario (1932) & $\mathrm{p}$-valor $<0,01 \%$ & $\mathrm{p}$-valor $<0,01 \%$ & $\mathrm{p}$-valor $<0,01 \%$ & $\mathrm{p}$-valor $<0,01 \%$ \\
\hline Brunt (1932) & $\mathrm{p}$-valor $<0,01 \%$ & $\mathrm{p}$-valor $<0,01 \%$ & $\mathrm{p}$-valor $<0,01 \%$ & $\mathrm{p}$-valor $<0,01 \%$ \\
\hline Ramanathan \& Desai (1932) & $\mathrm{p}$-valor $<0,01 \%$ & $\mathrm{p}$-valor $<0,01 \%$ & $\mathrm{p}$-valor $<0,01 \%$ & $\mathrm{p}$-valor $<0,01 \%$ \\
\hline Eckel (1934) & $\mathrm{p}$-valor $<0,01 \%$ & $\mathrm{p}$-valor $<0,01 \%$ & $\mathrm{p}$-valor $<0,01 \%$ & $\mathrm{p}$-valor $<0,01 \%$ \\
\hline Raman (1935) & $\mathrm{p}$-valor $<0,01 \%$ & $\mathrm{p}$-valor $<0,01 \%$ & $\mathrm{p}$-valor $<0,01 \%$ & $\mathrm{p}$-valor $<0,01 \%$ \\
\hline Elsasser (1942) & $\mathrm{p}$-valor $<0,01 \%$ & $\mathrm{p}$-valor $<0,01 \%$ & $\mathrm{p}$-valor $<0,01 \%$ & $\mathrm{p}$-valor $<0,01 \%$ \\
\hline Lutherstein \& Chednovsky (1946) & $\mathrm{p}$-valor $<0,01 \%$ & $\mathrm{p}$-valor $<0,01 \%$ & $\mathrm{p}$-valor $<0,01 \%$ & $\mathrm{p}$-valor $<0,01 \%$ \\
\hline Chumanova (1947) & $\mathrm{p}$-valor $<0,01 \%$ & $\mathrm{p}$-valor $<0,01 \%$ & $\mathrm{p}$-valor $<0,01 \%$ & $\mathrm{p}$-valor $<0,01 \%$ \\
\hline Berland \& Berland (1952) & $\mathrm{p}$-valor $<0,01 \%$ & $\mathrm{p}$-valor $<0,01 \%$ & $\mathrm{p}$-valor $<0,01 \%$ & $\mathrm{p}$-valor $<0,01 \%$ \\
\hline Anderson (1954) & $\mathrm{p}$-valor $<0,01 \%$ & $\mathrm{p}$-valor $<0,01 \%$ & $\mathrm{p}$-valor $<0,01 \%$ & $\mathrm{p}$-valor $<0,01 \%$ \\
\hline De Coster \& Shuepp (1957) & $\mathrm{p}$-valor $<0,01 \%$ & $\mathrm{p}$-valor $<0,01 \%$ & $\mathrm{p}$-valor $<0,01 \%$ & $\mathrm{p}$-valor $<0,01 \%$ \\
\hline Gross \& Brooks (1957) & $\mathrm{p}$-valor $<0,01 \%$ & $\mathrm{p}$-valor $<0,01 \%$ & $\mathrm{p}$-valor $<0,01 \%$ & $\mathrm{p}$-valor $<0,01 \%$ \\
\hline Bliss (1961) & $\mathrm{p}$-valor $<0,01 \%$ & $\mathrm{p}$-valor $<0,01 \%$ & $\mathrm{p}$-valor $<0,01 \%$ & $\mathrm{p}$-valor $<0,01 \%$ \\
\hline
\end{tabular}

continua... 
Tabela 3: continuação...

\begin{tabular}{|c|c|c|c|c|}
\hline Modelo & Shapiro-Wilk & Anderson-Darling & Lilliefors & Jarque-Bera \\
\hline Efimova (1961) & p-valor $<0,01 \%$ & $\mathrm{p}$-valor $<0,01 \%$ & p-valor $<0,01 \%$ & p-valor $<0,01 \%$ \\
\hline Harshunova (1961) mod1 & $\mathrm{p}$-valor $<0,01 \%$ & p-valor $<0,01 \%$ & p-valor $<0,01 \%$ & p-valor $<0,01 \%$ \\
\hline Harshunova (1961) mod2 & p-valor $<0,01 \%$ & $\mathrm{p}$-valor $<0,01 \%$ & p-valor $<0,01 \%$ & p-valor $<0,01 \%$ \\
\hline Monteith (1961) & p-valor $<0,01 \%$ & p-valor $<0,01 \%$ & p-valor $<0,01 \%$ & p-valor $<0,01 \%$ \\
\hline Swinbank (1963) - modelo 1 & p-valor $<0,01 \%$ & p-valor $<0,01 \%$ & p-valor $<0,01 \%$ & p-valor $<0,01 \%$ \\
\hline Swinbank (1963) - modelo 2 & $\mathrm{p}$-valor $<0,01 \%$ & $\mathrm{p}$-valor $<0,01 \%$ & $\mathrm{p}$-valor $<0,01 \%$ & p-valor $<0,01 \%$ \\
\hline Swinbank (1963) - modelo 3 & $\mathrm{p}$-valor $<0,01 \%$ & $\mathrm{p}$-valor $<0,01 \%$ & $\mathrm{p}$-valor $<0,01 \%$ & $\mathrm{p}$-valor $<0,01 \%$ \\
\hline Martin \& Palmer (1964) & p-valor $<0,01 \%$ & $\mathrm{p}$-valor $<0,01 \%$ & p-valor $<0,01 \%$ & p-valor $<0,01 \%$ \\
\hline Sellers (1965) & p-valor $<0,01 \%$ & $\mathrm{p}$-valor $<0,01 \%$ & $\mathrm{p}$-valor $<0,01 \%$ & p-valor $<0,01 \%$ \\
\hline Marshunova (1966) & p-valor $<0,01 \%$ & p-valor $<0,01 \%$ & p-valor $<0,01 \%$ & p-valor $<0,01 \%$ \\
\hline Idso \& Jackson (1969) & $\mathrm{p}$-valor $<0,01 \%$ & p-valor $<0,01 \%$ & p-valor $<0,01 \%$ & p-valor $<0,01 \%$ \\
\hline Deacon (1970) & p-valor $<0,01 \%$ & p-valor $<0,01 \%$ & p-valor $<0,01 \%$ & p-valor $<0,01 \%$ \\
\hline Viswanadham \& R. (1970) Madras & p-valor $<0,01 \%$ & p-valor $<0,01 \%$ & p-valor $<0,01 \%$ & p-valor $<0,01 \%$ \\
\hline Viswanadham \& R. (1970) Nagpur & $\mathrm{p}$-valor $<0,01 \%$ & p-valor $<0,01 \%$ & p-valor $<0,01 \%$ & p-valor $<0,01 \%$ \\
\hline Viswanadham \& R. (1970) Waltair & $\mathrm{p}$-valor $<0,01 \%$ & p-valor $<0,01 \%$ & $\mathrm{p}$-valor $<0,01 \%$ & p-valor $<0,01 \%$ \\
\hline Zillman (1972) & $\mathrm{p}$-valor $<0,01 \%$ & $\mathrm{p}$-valor $<0,01 \%$ & $\mathrm{p}$-valor $<0,01 \%$ & p-valor $<0,01 \%$ \\
\hline Maykut \& Church (1973) & p-valor $<0,01 \%$ & p-valor $<0,01 \%$ & p-valor $<0,01 \%$ & p-valor $<0,01 \%$ \\
\hline Brutsaert (1975) & $\mathrm{p}$-valor $<0,01 \%$ & p-valor $<0,01 \%$ & p-valor $<0,01 \%$ & p-valor $<0,01 \%$ \\
\hline Clark \& Allen (1978) & p-valor $<0,01 \%$ & p-valor $<0,01 \%$ & p-valor $<0,01 \%$ & p-valor $<0,01 \%$ \\
\hline Marks \& Dozier (1979) & p-valor $<0,01 \%$ & p-valor $<0,01 \%$ & p-valor $<0,01 \%$ & p-valor $<0,01 \%$ \\
\hline Satterlund (1979) & p-valor $<0,01 \%$ & $\mathrm{p}$-valor $<0,01 \%$ & p-valor $<0,01 \%$ & p-valor $<0,01 \%$ \\
\hline Idso (1981) & $\mathrm{p}$-valor $<0,01 \%$ & p-valor $<0,01 \%$ & p-valor $<0,01 \%$ & p-valor $<0,01 \%$ \\
\hline Ohmura (1981) & $\mathrm{p}$-valor $<0,01 \%$ & $\mathrm{p}$-valor $<0,01 \%$ & $\mathrm{p}$-valor $<0,01 \%$ & $\mathrm{p}$-valor $<0,01 \%$ \\
\hline Andreas \& Ackley (1982) & p-valor $<0,01 \%$ & p-valor $<0,01 \%$ & $\mathrm{p}$-valor $<0,01 \%$ & p-valor $<0,01 \%$ \\
\hline Berdhal \& Fromberg (1982) mod1 & p-valor $<0,01 \%$ & p-valor $<0,01 \%$ & p-valor $<0,01 \%$ & p-valor $<0,01 \%$ \\
\hline Berdhal \& Fromberg (1982) mod2 & $\mathrm{p}$-valor $<0,01 \%$ & $\mathrm{p}$-valor $<0,01 \%$ & p-valor $<0,01 \%$ & p-valor $<0,01 \%$ \\
\hline Centeno (1982) & p-valor $<0,01 \%$ & $\mathrm{p}$-valor $<0,01 \%$ & p-valor $<0,01 \%$ & p-valor $<0,01 \%$ \\
\hline Sugita e Brutsaert (1983) & p-valor $<0,01 \%$ & $\mathrm{p}$-valor $<0,01 \%$ & p-valor $<0,01 \%$ & p-valor $<0,01 \%$ \\
\hline Berdhal \& Martin (1984) & $\mathrm{p}$-valor $<0,01 \%$ & p-valor $<0,01 \%$ & p-valor $<0,01 \%$ & p-valor $<0,01 \%$ \\
\hline Berger et al. (1984) mod1 & p-valor $<0,01 \%$ & $\mathrm{p}$-valor $<0,01 \%$ & $\mathrm{p}$-valor $<0,01 \%$ & p-valor $<0,01 \%$ \\
\hline Berger et al. (1984) mod2 & p-valor $<0,01 \%$ & p-valor $<0,01 \%$ & $\mathrm{p}$-valor $<0,01 \%$ & p-valor $<0,01 \%$ \\
\hline Keding (1989) & $\mathrm{p}$-valor $<0,01 \%$ & $\mathrm{p}$-valor $<0,01 \%$ & $\mathrm{p}$-valor $<0,01 \%$ & p-valor $<0,01 \%$ \\
\hline FAO (1990) & $\mathrm{p}$-valor $<0,01 \%$ & $\mathrm{p}$-valor $<0,01 \%$ & p-valor $<0,01 \%$ & p-valor $<0,01 \%$ \\
\hline Kondo et al. (1990) & $\mathrm{p}$-valor $<0,01 \%$ & $\mathrm{p}$-valor $<0,01 \%$ & p-valor $<0,01 \%$ & p-valor $<0,01 \%$ \\
\hline Heitor et al. (1991) & $\mathrm{p}$-valor $<0,01 \%$ & p-valor $<0,01 \%$ & p-valor $<0,01 \%$ & p-valor $<0,01 \%$ \\
\hline Korsgaard et al. (1991) & p-valor $<0,01 \%$ & p-valor $<0,01 \%$ & p-valor $<0,01 \%$ & p-valor $<0,01 \%$ \\
\hline Garratt (1992) & p-valor $<0,01 \%$ & p-valor $<0,01 \%$ & p-valor $<0,01 \%$ & p-valor $<0,01 \%$ \\
\hline König-Langlo \& Augstein (1994) & p-valor $<0,01 \%$ & $\mathrm{p}$-valor $<0,01 \%$ & p-valor $<0,01 \%$ & p-valor $<0,01 \%$ \\
\hline Konzelmann et al (1994) & $\mathrm{p}$-valor $<0,01 \%$ & p-valor $<0,01 \%$ & p-valor $<0,01 \%$ & p-valor $<0,01 \%$ \\
\hline Prata (1996) & $\mathrm{p}$-valor $<0,01 \%$ & $\mathrm{p}$-valor $<0,01 \%$ & $\mathrm{p}$-valor $<0,01 \%$ & p-valor $<0,01 \%$ \\
\hline Greuell et al. (1997) & p-valor $<0,01 \%$ & p-valor $<0,01 \%$ & p-valor $<0,01 \%$ & p-valor $<0,01 \%$ \\
\hline Dilley \& O'Brien (1998) & $\mathrm{p}$-valor $<0,01 \%$ & $\mathrm{p}$-valor $<0,01 \%$ & p-valor $<0,01 \%$ & p-valor $<0,01 \%$ \\
\hline Crawford \& Duchon (1999) & $\mathrm{p}$-valor $<0,01 \%$ & $\mathrm{p}$-valor $<0,01 \%$ & p-valor $<0,01 \%$ & p-valor $<0,01 \%$ \\
\hline Niemelä et al. (2001) & p-valor $<0,01 \%$ & p-valor $<0,01 \%$ & p-valor $<0,01 \%$ & p-valor $<0,01 \%$ \\
\hline Zapadka et al (2001) - modelo 1 & $\mathrm{p}$-valor $<0,01 \%$ & p-valor $<0,01 \%$ & p-valor $<0,01 \%$ & p-valor $<0,01 \%$ \\
\hline Zapadka et al (2007) - modelo 2 & p-valor $<0,01 \%$ & p-valor $<0,01 \%$ & p-valor $<0,01 \%$ & p-valor $<0,01 \%$ \\
\hline Klok \& Oerlemans (2002) & $\mathrm{p}$-valor $<0,01 \%$ & p-valor $<0,01 \%$ & p-valor $<0,01 \%$ & p-valor $<0,01 \%$ \\
\hline Sridhar et al. (2002) & $\mathrm{p}$-valor $<0,01 \%$ & p-valor $<0,01 \%$ & $\mathrm{p}$-valor $<0,01 \%$ & p-valor $<0,01 \%$ \\
\hline Iziomon et al (2003) mod 1 & p-valor $<0,01 \%$ & p-valor $<0,01 \%$ & p-valor $<0,01 \%$ & p-valor $<0,01 \%$ \\
\hline
\end{tabular}

continua... 
Tabela 3: continuação...

\begin{tabular}{|c|c|c|c|c|}
\hline Modelo & Shapiro-Wilk & Anderson-Darling & Lilliefors & Jarque-Bera \\
\hline Iziomon et al. (2003) $\bmod 2$ & p-valor $<0,01 \%$ & $\mathrm{p}$-valor $<0,01 \%$ & $\mathrm{p}$-valor $<0,01 \%$ & p-valor $<0,01 \%$ \\
\hline Iziomon et al. (2003) $\bmod 3$ & p-valor $<0,01 \%$ & $\mathrm{p}$-valor $<0,01 \%$ & $\mathrm{p}$-valor $<0,01 \%$ & p-valor $<0,01 \%$ \\
\hline Golaka \& Excell (2004) mod1 & p-valor $<0,01 \%$ & p-valor $<0,01 \%$ & $\mathrm{p}$-valor $<0,01 \%$ & p-valor $<0,01 \%$ \\
\hline Golaka \& Excell (2004) mod2 & p-valor $<0,01 \%$ & p-valor $<0,01 \%$ & $\mathrm{p}$-valor $<0,01 \%$ & p-valor $<0,01 \%$ \\
\hline Golaka \& Excell (2004) mod3 & $\mathrm{p}$-valor $<0,01 \%$ & $\mathrm{p}$-valor $<0,01 \%$ & $\mathrm{p}$-valor $<0,01 \%$ & p-valor $<0,01 \%$ \\
\hline Golaka \& Excell (2004) mod4 & p-valor $<0,01 \%$ & p-valor $<0,01 \%$ & $\mathrm{p}$-valor $<0,01 \%$ & p-valor $<0,01 \%$ \\
\hline Duarte et al. (2006) & $\mathrm{p}$-valor $<0,01 \%$ & $\mathrm{p}$-valor $<0,01 \%$ & $\mathrm{p}$-valor $<0,01 \%$ & p-valor $<0,01 \%$ \\
\hline Jin et al. (2006) & $\mathrm{p}$-valor $<0,01 \%$ & $\mathrm{p}$-valor $<0,01 \%$ & $\mathrm{p}$-valor $<0,01 \%$ & p-valor $<0,01 \%$ \\
\hline Silva et al. (2006) & p-valor $<0,01 \%$ & p-valor $<0,01 \%$ & $\mathrm{p}$-valor $<0,01 \%$ & $\mathrm{p}$-valor $<0,01 \%$ \\
\hline Silva et al. (2006) ${ }_{2000}$ & $\mathrm{p}$-valor $<0,01 \%$ & $\mathrm{p}$-valor $<0,01 \%$ & $\mathrm{p}$-valor $<0,01 \%$ & $\mathrm{p}$-valor $<0,01 \%$ \\
\hline Lhomme et al. (2007) & p-valor $<0,01 \%$ & p-valor $<0,01 \%$ & $\mathrm{p}$-valor $<0,01 \%$ & p-valor $<0,01 \%$ \\
\hline Kruk et al. (2009) & p-valor $<0,01 \%$ & $\mathrm{p}$-valor $<0,01 \%$ & $\mathrm{p}$-valor $<0,01 \%$ & p-valor $<0,01 \%$ \\
\hline Bárbaro et al. (2010) & $\mathrm{p}$-valor $<0,01 \%$ & p-valor $<0,01 \%$ & $\mathrm{p}$-valor $<0,01 \%$ & p-valor $<0,01 \%$ \\
\hline
\end{tabular}

Mesmo utilizando os 107 modelos com seus parâmetros originais pode-se observar que os resultados obtidos não foram comprometidos visto que a maioria dos modelos tiveram um ótimo desempenho.

Os modelos utilizados para o cálculo da irradiância de ondas longas em condições de céu claro, parcialmente nublado e nublado foram categorizados e se enquadraram em uma das oito categorias propostas de acordo com os seus parâmetros. A tabela 4 mostra a avaliação dos oitenta (80) modelos propostos para as condições de céu claro, categorizados e classificados em ordem decrescente em função de suas respectivas estatísticas $c, d, r$ e $R^{2}$ e crescente em termos de ERM, EMA e EMQ.

Tabela 4 Avaliação dos modelos de estimativa da irradiância de ondas longas para condições de céu claro (Ldcs)

\begin{tabular}{|c|c|c|c|c|c|c|c|c|c|c|}
\hline Modelo & Ano & $\mathrm{R}^{2}$ & $\mathrm{r}$ & $\mathrm{d}$ & $\begin{array}{c}\text { EMQ } \\
\%\end{array}$ & $\begin{array}{l}\text { EMA } \\
\text { W.m }{ }^{-2}\end{array}$ & $\underset{\%}{\text { ERM }}$ & c & Interpretação & Categoria \\
\hline $\begin{array}{l}\text { Viswanadham \& R. (1970) } \\
\text { Madras }\end{array}$ & 1970 & 0,9724 & 0,9861 & 0,9937 & 1,3619 & 3,5096 & 0,0011 & 0,9799 & о́тімо & 3 \\
\hline Satterlund (1979) & 1979 & 0,9705 & 0,9851 & 0,9925 & 1,4924 & 4,1351 & 0,6309 & 0,9778 & о́тімо & 3 \\
\hline Marshunova (1966) & 1966 & 0,9712 & 0,9855 & 0,9916 & 1,5612 & 4,3461 & 0,5841 & 0,9772 & о́тIMо & 3 \\
\hline $\begin{array}{l}\text { Viswanadham \& R. (1970) } \\
\text { Waltair }\end{array}$ & 1970 & 0,9688 & 0,9843 & 0,9927 & 1,4845 & 4,2613 & 0,3405 & 0,9771 & о́тімо & 3 \\
\hline Berger et al. (1984) mod1 & 1984 & 0,9723 & 0,9861 & 0,9904 & 1,6722 & 4,7368 & 0,8254 & 0,9766 & о́тімо & 3 \\
\hline FAO (1990) & 1990 & 0,9721 & 0,9860 & 0,9886 & 1,8292 & 5,3170 & 1,0668 & 0,9747 & о́тIмо & 3 \\
\hline Konzelmann et al (1994) & 1994 & 0,9628 & 0,9812 & 0,9911 & 1,5853 & 4,3312 & 0,2676 & 0,9725 & о́тімо & 3 \\
\hline Gross \& Brooks (1957) & 1957 & 0,9722 & 0,9860 & 0,9858 & 2,0336 & 6,2685 & 1,3557 & 0,9720 & о́TIMO & 3 \\
\hline $\begin{array}{l}\text { Viswanadham \& R. (1970) } \\
\text { Nagpur }\end{array}$ & 1970 & 0,9709 & 0,9853 & 0,9854 & 2,1095 & 6,9894 & 1,7028 & 0,9710 & о́тімо & 3 \\
\hline Kondo et al. (1990) & 1990 & 0,9596 & 0,9796 & 0,9903 & 1,6819 & 5,2421 & 0,6293 & 0,9701 & о́тімо & 6 \\
\hline Prata (1996) & 1996 & 0,9607 & 0,9802 & 0,9887 & 1,8060 & 4,4849 & 0,6810 & 0,9691 & о́тімо & 6 \\
\hline Berger et al. (1984) mod2 & 1984 & 0,9640 & 0,9818 & 0,9854 & 2,0379 & 6,2337 & 1,1814 & 0,9675 & о́тімо & 4 \\
\hline Brutsaert (1975) & 1975 & 0,9553 & 0,9774 & 0,9889 & 1,7929 & 4,5384 & 0,3701 & 0,9665 & о́тімо & 3 \\
\hline $\begin{array}{l}\text { Lutherstein \& Chednovsky } \\
\text { (1946) }\end{array}$ & 1946 & 0,9552 & 0,9773 & 0,9863 & 2,0433 & 5,3959 & 0,8561 & 0,9640 & о́тімо & 3 \\
\hline Greuell et al. (1997) & 1997 & 0,9628 & 0,9812 & 0,9802 & 2,3696 & 7,3363 & 1,6169 & 0,9617 & о́тімо & 3 \\
\hline $\begin{array}{l}\text { Berdhal \& Fromberg (1982) } \\
\text { mod2 }\end{array}$ & 1982 & 0,9623 & 0,9810 & 0,9803 & 2,4408 & 7,2263 & 1,7028 & 0,9616 & о́тімо & 4 \\
\hline Clark \& Allen (1978) & 1978 & 0,9514 & 0,9754 & 0,9799 & 2,3800 & 7,3872 & 1,4282 & 0,9558 & о́тIмо & 4 \\
\hline
\end{tabular}


Tabela 4 continuação...

\begin{tabular}{|c|c|c|c|c|c|c|c|c|c|c|}
\hline Modelo & Ano & $\mathrm{R}^{2}$ & $\mathrm{r}$ & $\mathrm{d}$ & $\begin{array}{c}\text { EMQ } \\
\%\end{array}$ & $\begin{array}{l}\text { EMA } \\
\text { W.m }{ }^{-2}\end{array}$ & $\begin{array}{c}\text { ERM } \\
\%\end{array}$ & $\mathrm{c}$ & Interpretação & Categoria \\
\hline Berland \& Berland (1952) & 1952 & 0,9650 & 0,9823 & 0,9692 & 3,1372 & 10,7389 & 2,8135 & 0,9521 & о́тімо & 3 \\
\hline Korsgaard et al. (1991) & 1991 & 0,9684 & 0,9841 & 0,9660 & 3,2932 & 11,4500 & 3,0548 & 0,9506 & о́TIMO & 3 \\
\hline $\begin{array}{l}\text { Berdhal \& Fromberg (1982) } \\
\text { mod1 }\end{array}$ & 1982 & 0,9307 & 0,9647 & 0,9836 & 2,1202 & 5,7310 & 0,4519 & 0,9489 & о́тімо & 4 \\
\hline Berdhal \& Martin (1984) & 1984 & 0,9605 & 0,9801 & 0,9674 & 3,1529 & 10,1089 & 2,5626 & 0,9481 & о́тімо & 3 \\
\hline Sellers (1965) & 1965 & 0,9702 & 0,9850 & 0,9587 & 3,5213 & 11,9919 & 3,0801 & 0,9443 & о́тімо & 3 \\
\hline Kruk et al. (2009) & 2009 & 0,9171 & 0,9577 & 0,9801 & 2,4542 & 7,0403 & 0,0435 & 0,9386 & о́тімо & 3 \\
\hline Iziomon et al (2003) mod 1 & 2003 & 0,9556 & 0,9775 & 0,9580 & 3,5130 & 11,4473 & 2,9059 & 0,9365 & о́тімо & 3 \\
\hline Iziomon et al. (2003) mod2 & 2003 & 0,9556 & 0,9775 & 0,9580 & 3,5130 & 11,4473 & 2,9059 & 0,9365 & о́тімо & 3 \\
\hline Chumanova (1947) & 1947 & 0,9721 & 0,9860 & 0,9494 & 3,8905 & 13,5019 & 3,4871 & 0,9361 & о́тімо & 3 \\
\hline Dines \& Dines (1927) & 1927 & 0,9516 & 0,9755 & 0,9593 & 3,5513 & 11,3905 & 2,9246 & 0,9358 & о́тімо & 3 \\
\hline Monteith (1961) & 1961 & 0,9516 & 0,9755 & 0,9593 & 3,5513 & 11,3905 & 2,9246 & 0,9358 & о́тімо & 3 \\
\hline Efimova (1961) & 1961 & 0,9650 & 0,9823 & 0,9473 & 4,1883 & 14,8031 & 3,9721 & 0,9305 & о́тімо & 3 \\
\hline Jin et al. (2006) & 2006 & 0,9606 & 0,9801 & 0,9476 & 4,7685 & 15,1172 & 3,8357 & 0,9287 & о́TIMO & 3 \\
\hline Swinbank (1963) - modelo 1 & 1963 & 0,9042 & 0,9509 & 0,9625 & 3,9399 & 13,3200 & 2,1677 & 0,9152 & о́тімо & 3 \\
\hline Harshunova (1961) mod2 & 1961 & 0,9042 & 0,9509 & 0,9625 & 3,9399 & 13,3188 & 2,1677 & 0,9152 & о́тімо & 3 \\
\hline Swinbank (1963) - modelo 3 & 1963 & 0,9042 & 0,9509 & 0,9621 & 3,9626 & 13,3922 & 2,2200 & 0,9148 & о́тімо & 1 \\
\hline Idso \& Jackson (1969) & 1969 & 0,8965 & 0,9468 & 0,9645 & 3,8856 & 13,0425 & 1,4111 & 0,9132 & о́тімо & 1 \\
\hline Deacon (1970) & 1970 & 0,9034 & 0,9505 & 0,9549 & 4,3320 & 14,6032 & 2,9194 & 0,9076 & о́тімо & 5 \\
\hline De Coster \& Shuepp (1957) & 1957 & 0,9686 & 0,9842 & 0,9148 & 5,4533 & 19,9100 & 5,3570 & 0,9003 & о́TIMO & 3 \\
\hline Niemelä et al. (2001) & 2001 & 0,9345 & 0,9667 & 0,9276 & 5,0746 & 17,2502 & 4,5383 & 0,8967 & о́тімо & 3 \\
\hline Ångström (1918) & 1918 & 0,9028 & 0,9502 & 0,9385 & 4,1658 & 13,5060 & 3,1674 & 0,8917 & о́тімо & 3 \\
\hline Brunt (1932) & 1932 & 0,9479 & 0,9736 & 0,9155 & 5,2209 & 18,1162 & 4,7568 & 0,8913 & о́тімо & 3 \\
\hline Swinbank (1963) - modelo 2 & 1963 & 0,9116 & 0,9548 & 0,9307 & 5,3537 & 17,9867 & 4,5943 & 0,8886 & о́тімо & 1 \\
\hline Robitsch (1926) & 1926 & 0,8497 & 0,9218 & 0,9522 & 4,0547 & 11,0960 & 1,5056 & 0,8777 & о́тімо & 3 \\
\hline Golaka \& Excell (2004) mod1 & 2004 & 0,8701 & 0,9328 & 0,9410 & 4,0803 & 13,3044 & 3,4202 & 0,8777 & о́TIMO & 1 \\
\hline Lhomme et al. (2007) & 2007 & 0,9553 & 0,9774 & 0,8976 & 5,6279 & 19,8980 & 5,1909 & 0,8773 & о́тімо & 3 \\
\hline Iziomon et al. (2003) mod3 & 2003 & 0,9281 & 0,9634 & 0,8935 & 5,8254 & 20,0699 & 5,2532 & 0,8608 & о́тімо & 3 \\
\hline Bárbaro et al. (2010) & 2010 & 0,9439 & 0,9715 & 0,8820 & 6,2392 & 22,0800 & 5,7777 & 0,8569 & о́тімо & 3 \\
\hline Asklef (1928) & 1928 & 0,9126 & 0,9553 & 0,8842 & 6,3245 & 21,6616 & 5,7223 & 0,8447 & MUITO вом & 3 \\
\hline Elsasser (1942) & 1942 & 0,9527 & 0,9761 & 0,8654 & 6,4922 & 23,2249 & 6,0470 & 0,8447 & MUITO вом & 3 \\
\hline Boutario (1932) & 1932 & 0,9720 & 0,9859 & 0,8468 & 7,1475 & 26,1087 & 6,8524 & 0,8348 & MUITO вом & 3 \\
\hline Heitor et al. (1991) & 1991 & 0,9713 & 0,9855 & 0,8431 & 7,2645 & 26,5500 & 6,9731 & 0,8309 & MUITO вом & 3 \\
\hline Sridhar et al. (2002) & 2002 & 0,9625 & 0,9811 & 0,8384 & 7,2419 & 26,2719 & 6,8643 & 0,8225 & MUITO вом & 3 \\
\hline Harshunova (1961) mod1 & 1961 & 0,9723 & 0,9861 & 0,8296 & 7,6133 & 27,8869 & 7,3224 & 0,8181 & мUITO вом & 3 \\
\hline Dilley \& O'Brien (1998) & 1998 & 0,8206 & 0,9059 & 0,8772 & 5,7032 & 18,0457 & 4,5700 & 0,7946 & MUITO вом & 6 \\
\hline Idso (1981) & 1981 & 0,7847 & 0,8858 & 0,8895 & 5,7582 & 19,4301 & 4,9459 & 0,7880 & MUITO BOM & 3 \\
\hline Zapadka et al (2001) - modelo 2 & 2001 & 0,9725 & 0,9862 & 0,7979 & 8,5051 & 31,2912 & 8,2288 & 0,7869 & MUITO вом & 3 \\
\hline Klok \& Oerlemans (2002) & 2002 & 0,9624 & 0,9810 & 0,8007 & 8,2734 & 30,2180 & 7,9138 & 0,7855 & MUITO BOM & 3 \\
\hline Ohmura (1981) & 1981 & 0,9403 & 0,9697 & 0,8092 & 8,5995 & 31,3038 & 8,2928 & 0,7847 & MUITO вOм & 1 \\
\hline Keding (1989) & 1989 & 0,9000 & 0,9487 & 0,8020 & 8,8019 & 32,0902 & 8,6758 & 0,7609 & MUITO вом & 3 \\
\hline Centeno (1982) & 1982 & 0,9619 & 0,9808 & 0,7567 & 9,5239 & 34,9881 & 9,1848 & 0,7422 & ВОМ & 5 \\
\hline Garratt (1992) & 1992 & 0,9595 & 0,9795 & 0,7337 & 10,1955 & 37,5139 & 9,8540 & 0,7187 & вом & 3 \\
\hline Eckel (1934) & 1934 & 0,9453 & 0,9723 & 0,7143 & 11,3453 & 41,8687 & 11,0727 & 0,6945 & BOM & 3 \\
\hline
\end{tabular}


Tabela 4 continuação...

\begin{tabular}{|c|c|c|c|c|c|c|c|c|c|c|}
\hline Modelo & Ano & $\mathrm{R}^{2}$ & $\mathrm{r}$ & d & $\begin{array}{c}\text { EMQ } \\
\%\end{array}$ & $\begin{array}{l}\text { EMA } \\
\text { W.m. } \\
\end{array}$ & $\begin{array}{c}\text { ERM } \\
\%\end{array}$ & c & Interpretação & Categoria \\
\hline $\begin{array}{l}\text { König-Langlo \& Augstein } \\
\text { (1994) }\end{array}$ & 1994 & 0,9000 & 0,9487 & 0,7231 & 10,2897 & 37,2609 & 9,7519 & 0,6860 & BOM & 1 \\
\hline Andreas \& Ackley (1982) & 1982 & 0,7342 & 0,8569 & 0,7989 & 7,9838 & 26,1419 & 6,7486 & 0,6845 & BOM & 3 \\
\hline Raman (1935) & 1935 & 0,9692 & 0,9845 & 0,6914 & 11,6720 & 43,2291 & 11,3836 & 0,6806 & BOM & 3 \\
\hline Bliss (1961) & 1961 & 0,9559 & 0,9777 & 0,6729 & 13,6691 & 51,3040 & 13,7456 & 0,6579 & BOM & 4 \\
\hline Ramanathan \& Desai (1932) & 1932 & 0,9721 & 0,9860 & 0,6055 & 15,1256 & 56,3347 & 14,8799 & 0,5970 & SOFRÍVEL & 3 \\
\hline Kimball (1918) & 1918 & 0,9426 & 0,9709 & 0,5948 & 15,9133 & 59,2057 & 15,6771 & 0,5775 & SOFRÍVEL & 3 \\
\hline Maykut \& Church (1973) & 1973 & 0,9000 & 0,9487 & 0,5993 & 14,8358 & 54,6757 & 14,3824 & 0,5685 & SOFRÍVEL & 1 \\
\hline $\begin{array}{l}\text { Zapadka et al (2001) - modelo } \\
1\end{array}$ & 2001 & 0,9341 & 0,9665 & 0,4895 & 20,8429 & 77,6147 & 20,4891 & 0,4731 & MAU & 3 \\
\hline Sugita e Brutsaert (1983) & 1983 & 0,9567 & 0,9781 & 0,3752 & 30,2451 & 113,1210 & 29,9333 & 0,3670 & PÉSSIMO & 3 \\
\hline Marks \& Dozier (1979) & 1979 & 0,9553 & 0,9774 & 0,3659 & 35,4324 & 133,1830 & 35,4649 & 0,3576 & PÉSSIMO & 8 \\
\hline Zillman (1972) & 1972 & 0,2901 & 0,5386 & 0,6489 & 13,5422 & 42,8473 & 7,4462 & 0,3495 & PÉSSIMO & 1 \\
\hline Martin \& Palmer (1964) & 1964 & 0,9002 & 0,9488 & 0,3548 & 32,1697 & 120,2150 & 31,7994 & 0,3366 & PÉSSIMO & 3 \\
\hline Anderson (1954) & 1954 & 0,9259 & 0,9622 & 0,3298 & 40,9160 & 153,2119 & 40,6946 & 0,3173 & PÉSSIMO & 3 \\
\hline Crawford \& Duchon (1999) & 1999 & 0,8619 & 0,9284 & 0,2983 & 45,5468 & 171,1499 & 45,6611 & 0,2770 & PÉSSIMO & 7 \\
\hline Duarte et al. (2006) & 2006 & 0,9595 & 0,9795 & 0,2547 & 48,4576 & 181,6486 & 48,1525 & 0,2495 & PÉSSIMO & 3 \\
\hline Silva et al. (2006) & 2006 & 0,9700 & 0,9849 & 0,2532 & 56,2386 & 211,4870 & 56,2718 & 0,2494 & PÉSSIMO & 3 \\
\hline Silva et al. (2006) 2000 & 2006 & 0,9720 & 0,9859 & 0,1416 & 108,1560 & 406,4310 & 108,0180 & 0,1396 & PÉSSIMO & 3 \\
\hline Golaka \& Excell (2004) mod4 & 2004 & $-1,3680$ & $\nexists$ & 0,7276 & 6,7361 & 22,0658 & 1,2970 & 0,0000 & PÉSSIMO & 3 \\
\hline Golaka \& Excell (2004) mod2 & 2004 & $-2,6320$ & $\nexists$ & 0,0032 & 8,7642 & 27,2504 & 0,2275 & 0,0000 & PÉSSIMO & 2 \\
\hline Golaka \& Excell (2004) mod3 & 2004 & $-2,1210$ & $\nexists$ & 0,5179 & 9,3046 & 29,1618 & 0,0682 & 0,0000 & PÉSSIMO & 3 \\
\hline
\end{tabular}

Nesta análise, podemos observar que o modelo (original) de estimativa da irradiância de ondas longas para condições de céu claro que apresentou os melhores índices estatísticos e, portanto, aquele que melhor se adapta às condições no cerrado Mato-grossense, para o período estudado, foi o modelo de Viswanadham \& Ramanadham (1970) com os seus coeficientes ajustados para
Madras na Índia.

A tabela 5 mostra a avaliação dos vinte e sete (27) modelos propostos para as condições de céu parcialmente nublado, categorizados e classificados em ordem decrescente em função de suas respectivas estatísticas $c, d, r$ e $R^{2}$ e crescente em termos de ERM, EMA e EMQ.

Tabela 5: Avaliação dos modelos de estimativa da irradiância de ondas longas para condições de céu parcialmente nublado $\left(L d_{c l d}\right)$

\begin{tabular}{|c|c|c|c|c|c|c|c|c|c|c|}
\hline Modelo & Ano & $\mathrm{R}^{2}$ & $\mathrm{r}$ & d & $\begin{array}{c}\text { EMQ } \\
\%\end{array}$ & $\begin{array}{l}\text { EMA } \\
\text { W.m }{ }^{-2}\end{array}$ & $\begin{array}{c}\text { ERM } \\
\%\end{array}$ & $\mathrm{c}$ & Interpretação & Categoria \\
\hline Idso \& Jackson (1969) & 1969 & 0,9263 & 0,9624 & 0,9749 & 2,9614 & 8,6486 & 0,9713 & 0,9383 & о́TIмо & 1 \\
\hline $\begin{array}{l}\text { Crawford and Duchon } \\
\text { (1999) }\end{array}$ & 1999 & 0,8497 & 0,9218 & 0,9647 & 2,9402 & 9,5366 & 0,7749 & 0,8893 & о́тімо & 7 \\
\hline Lhomme et al. (2007) & 2007 & 0,8773 & 0,9366 & 0,9488 & 3,6680 & 12,4226 & 2,3301 & 0,8887 & о́TIмо & 3 \\
\hline Bignami et al. (1995) & 1995 & 0,9251 & 0,9618 & 0,9037 & 5,2692 & 19,3207 & 4,6435 & 0,8692 & ÓTIMo & 3 \\
\hline Staley and Jurica (1972) & 1972 & 0,8735 & 0,9346 & 0,9226 & 4,5486 & 15,9322 & 4,1373 & 0,8623 & ÓTIMO & 8 \\
\hline $\begin{array}{l}\text { Bilbao \& de Miguel } \\
\text { (2007) mod1 }\end{array}$ & 2007 & 0,8559 & 0,9251 & 0,9168 & 5,0599 & 17,5002 & 4,0904 & 0,8481 & MUITO BOM & 3 \\
\hline Aubinet (1994) - mod 01 & 1994 & 0,7775 & 0,8818 & 0,9497 & 3,5892 & 11,8223 & 0,0914 & 0,8374 & MUITO вOM & 1 \\
\hline
\end{tabular}

continua... 
Tabela 5: continuação...

\begin{tabular}{|l|c|c|c|c|c|c|c|c|c|c|}
\hline \multicolumn{1}{|c|}{ Modelo } & Ano & $\mathrm{R}^{2}$ & $\mathrm{r}$ & $\mathrm{d}$ & $\begin{array}{c}\text { EMQ } \\
\%\end{array}$ & $\begin{array}{c}\text { EMA } \\
\text { W.m }{ }^{-2}\end{array}$ & $\begin{array}{c}\text { ERM } \\
\%\end{array}$ & $\mathrm{c}$ & Interpretação & Categoria \\
\hline $\begin{array}{l}\text { Parkinson \& Washington } \\
\text { (1979) }\end{array}$ & 1979 & 0,9041 & 0,9508 & 0,8704 & 7,2622 & 25,8323 & 6,3297 & 0,8277 & MUITO BOM & 1 \\
\hline Zapadka et al. (2007) & 2007 & 0,8886 & 0,9427 & 0,8419 & 6,9379 & 26,0741 & 6,2473 & 0,7936 & MUITO BOM & 3 \\
\hline Aubinet (1994) - mod 04 & 1994 & 0,7098 & 0,8425 & 0,9384 & 3,8601 & 13,0006 & 0,8362 & 0,7906 & MUITO BOM & 3 \\
\hline $\begin{array}{l}\text { Bilbao \& de Miguel } \\
\text { (2007) mod4 }\end{array}$ & 2007 & 0,8928 & 0,9449 & 0,7968 & 0,2486 & 0,0290 & 0,0087 & 0,7529 & MUITO BOM & 1 \\
\hline Croley (1989) & 1989 & 0,8028 & 0,8960 & 0,8202 & 7,9525 & 28,6995 & 7,1714 & 0,7349 & BOM & 3 \\
\hline Kimball et al. (1982) & 1982 & 0,7968 & 0,8926 & 0,8159 & 7,5440 & 28,2569 & 7,2204 & 0,7283 & BOM & 3 \\
\hline $\begin{array}{l}\text { Bilbao \& de Miguel } \\
\text { (2007) mod2 }\end{array}$ & 2007 & 0,8571 & 0,9258 & 0,7865 & 8,7684 & 33,6627 & 8,5011 & 0,7281 & BOM & 3 \\
\hline Andreas \& Ackley (1982) & 1982 & 0,6729 & 0,8203 & 0,8229 & 7,1173 & 23,9028 & 5,5397 & 0,6751 & BOM & 3 \\
\hline Marschunova (1961) & 1961 & 0,8645 & 0,9298 & 0,6614 & 12,7391 & 50,3114 & 12,6288 & 0,6150 & MEDIANO & 3 \\
\hline Augstein (1994) & 1994 & 0,8052 & 0,8973 & 0,6796 & 11,4340 & 44,0808 & 10,7045 & 0,6098 & MEDIANO & 1 \\
\hline $\begin{array}{l}\text { König - Langlo \& } \\
\text { Augstein (1994) }\end{array}$ & 1994 & 0,8052 & 0,8973 & 0,6796 & 11,4340 & 44,0808 & 10,7045 & 0,6098 & MEDIANO & 1 \\
\hline
\end{tabular}

Nesta análise, podemos observar que o modelo original de estimativa da irradiância de ondas longas para condições de céu parcialmente nublado que apresentou os melhores índices estatísticos e, portanto, aquele que melhor se adapta às condições no cerrado Mato-grossense, para o período estudado, foi o modelo de Idso \& Jackson (1969).

A tabela 6 mostra a avaliação dos vinte e sete (27) modelos propostos para as condições de céu nublado, categorizados e classificados em ordem decrescente em função de suas respectivas estatísticas $c, d, r e R^{2}$ e crescente em termos de ERM, EMA e EMQ.
Nesta análise, podemos observar que o modelo original de estimativa da irradiância de ondas longas para condições de céu nublado que apresentou os melhores índices estatísticos e, portanto, aquele que melhor se adapta às condições no cerrado Mato-grossense, para o período estudado, foi o modelo de Bignami et al. (1995).

As tabelas (7), (8) e (9) mostram os resultados estatísticos obtidos após a parametrização dos modelos que tiveram desempenho "ÓTIMO" e "MUITO BOM" nas condições de céu claro, céu parcialmente nublado e nublado no cerrado Mato-grossense.

Tabela 6: Avaliação dos modelos de estimativa da irradiância de ondas longas para condições de céu nublado $\left(L d_{c l d}\right)$

\begin{tabular}{|l|c|c|c|c|c|c|c|c|c|c|}
\hline \multicolumn{1}{|c|}{ Modelo } & Ano & $\mathrm{R}^{2}$ & $\mathrm{r}$ & $\mathrm{d}$ & $\begin{array}{c}\text { EMQ } \\
\%\end{array}$ & $\begin{array}{c}\text { EMA } \\
\text { W.m }\end{array}$ & $\begin{array}{c}\text { ERM } \\
\%\end{array}$ & $\mathrm{c}$ & Interpretação & Categoria \\
\hline Bignami et al. (1995) & 1995 & 0,9439 & 0,9715 & 0,9862 & 1,7950 & 3,9330 & 0,0059 & 0,9581 & ÓTIMO & 3 \\
\hline Idso \& Jackson (1969) & 1969 & 0,9142 & 0,9561 & 0,9391 & 4,2130 & 12,3342 & 3,1595 & 0,8979 & ÓTIMO & 1 \\
\hline Zapadka et al. (2007) & 2007 & 0,8027 & 0,8959 & 0,9324 & 3,9456 & 11,0071 & 2,4180 & 0,8354 & MUITO BOM & 3 \\
\hline Lhomme et al. (2007) & 2007 & 0,7912 & 0,8895 & 0,9373 & 3,9056 & 8,8181 & 1,3973 & 0,8337 & MUITO BOM & 3 \\
\hline Andreas \& Ackley (1982) & 1982 & 0,7794 & 0,8828 & 0,9045 & 4,8046 & 15,4632 & 3,3435 & 0,7985 & MUITO BOM & 3 \\
\hline $\begin{array}{l}\text { Crawford and Duchon } \\
\text { (1999) }\end{array}$ & 1999 & 0,8540 & 0,9241 & 0,8246 & 6,7107 & 24,1106 & 6,5066 & 0,7620 & MUITO BOM & 7 \\
\hline $\begin{array}{l}\text { Bilbao \& de Miguel (2007) } \\
\text { mod4 }\end{array}$ & 2007 & 0,9359 & 0,9674 & 0,7749 & 9,2969 & 33,8417 & 8,8713 & 0,7497 & BOM & 1 \\
\hline Staley and Jurica (1972) & 1972 & 0,8705 & 0,9330 & 0,7291 & 9,2509 & 34,4116 & 9,2302 & 0,6802 & BOM & 8 \\
\hline Aubinet (1994) - mod 04 & 1994 & 0,8895 & 0,9431 & 0,7191 & 9,5693 & 35,7728 & 9,5793 & 0,6782 & BOM & 3 \\
\hline $\begin{array}{l}\text { Parkinson \& Washington } \\
\text { (1979) }\end{array}$ & 1979 & 0,9241 & 0,9613 & 0,7007 & 11,6801 & 42,8102 & 11,2250 & 0,6736 & BOM & 1 \\
\hline
\end{tabular}


Tabela 6: continuação...

\begin{tabular}{|l|c|c|c|c|c|c|c|c|c|c|}
\hline \multicolumn{1}{|c|}{ Modelo } & Ano & $\mathrm{R}^{2}$ & $\mathrm{r}$ & $\mathrm{d}$ & $\begin{array}{c}\text { EMQ } \\
\%\end{array}$ & $\begin{array}{c}\text { EMA } \\
\mathrm{W} \cdot \mathrm{m}^{-2}\end{array}$ & $\begin{array}{c}\text { ERM } \\
\%\end{array}$ & $\mathrm{c}$ & Interpretação & Categoria \\
\hline $\begin{array}{l}\text { Bilbao \& de Miguel (2007) } \\
\text { mod1 }\end{array}$ & 2007 & 0,9384 & 0,9687 & 0,6535 & 12,8307 & 47,8040 & 12,5833 & 0,6330 & MEDIANO & 3 \\
\hline Kimball et al. (1982) & 1982 & 0,9261 & 0,9623 & 0,6495 & 11,9418 & 45,0915 & 12,0123 & 0,6251 & MEDIANO & 3 \\
\hline Aubinet (1994) - mod 01 & 1994 & 0,8178 & 0,9043 & 0,6881 & 10,3905 & 38,3719 & 10,2807 & 0,6223 & MEDIANO & 1 \\
\hline Augstein (1994) & 1994 & 0,5603 & 0,7485 & 0,7517 & 7,7413 & 26,9815 & 6,4084 & 0,5627 & SOFRÍVEL & 1 \\
\hline $\begin{array}{l}\text { König - Langlo \& Augstein } \\
\text { (1994) }\end{array}$ & 1994 & 0,5603 & 0,7485 & 0,7517 & 7,7413 & 26,9815 & 6,4084 & 0,5627 & SOFRÍVEL & 1 \\
\hline $\begin{array}{l}\text { Bilbao \& de Miguel (2007) } \\
\text { mod2 }\end{array}$ & 2007 & 0,9492 & 0,9743 & 0,5519 & 16,4228 & 61,9648 & 16,3799 & 0,5377 & SOFRÍVEL & 3 \\
\hline Croley (1989) & 1989 & 0,8872 & 0,9419 & 0,4840 & 20,1170 & 75,3249 & 19,8701 & 0,4558 & MAU & 3 \\
\hline Marschunova (1961) & 1961 & 0,9264 & 0,9625 & 0,4463 & 22,1278 & 83,5576 & 22,0874 & 0,4295 & MAU & 3 \\
\hline $\begin{array}{l}\text { Bilbao \& de Miguel (2007) } \\
\text { mod3 }\end{array}$ & 2007 & 0,8545 & 0,9244 & 0,4175 & 23,6382 & 88,9220 & 23,5171 & 0,3859 & PÉSSIMO & 3 \\
\hline Aubinet (1994) - mod 02 & 1994 & 0,3578 & 0,5982 & 0,6391 & 10,4975 & 37,9300 & 10,1631 & 0,3823 & PÉSSIMO & 3 \\
\hline Cho et al. (2008) & 2008 & 0,4627 & 0,6802 & 0,4119 & 27,4821 & 98,9897 & 26,3957 & 0,2802 & PÉSSIMO & 8 \\
\hline Brutsaert (1975) & 1975 & 0,9093 & 0,9536 & 0,2389 & 44,8153 & 169,4151 & 44,5906 & 0,2278 & PÉSSIMO & 3 \\
\hline Konzelmann et al. (1994) & 1994 & 0,2504 & 0,5004 & 0,3700 & 25,1374 & 93,8886 & 24,2114 & 0,1852 & PÉSSIMO & 1 \\
\hline Centeno (1982) & 1982 & 0,1297 & 0,3601 & 0,2144 & 48,3641 & 181,8206 & 47,5267 & 0,0772 & PÉSSIMO & 5 \\
\hline Aubinet (1994) - mod 03 & 1994 & $-0,6470$ & $\nexists$ & 0,6430 & 8,5180 & 29,3019 & 6,7054 & 0,0000 & PÉSSIMO & 2 \\
\hline Gabathuler et al. (2001) & 2001 & $-0,4610$ & $\nexists$ & 0,4633 & 17,1026 & 62,6250 & 16,9071 & 0,0000 & PÉSSIMO & 5 \\
\hline Krężel et al. (2008) & 2008 & $-0,6730$ & $\nexists$ & 0,1689 & 32,1398 & 107,7680 & 26,3575 & 0,0000 & PÉSSIMO & 3 \\
\hline
\end{tabular}

Tabela 7: Avaliação dos modelos parametrizados para a estimativa da irradiância de ondas longas no cerrado Mato-grossense nas condições de céu claro $\left(\mathrm{L}_{\mathrm{cs}}{ }^{*}\right)$.

\begin{tabular}{|l|c|c|c|c|c|c|c|c|c|c|}
\hline \multicolumn{1}{|c|}{ Modelo } & Ano & $\mathrm{R}^{2}$ & $\mathrm{r}$ & $\mathrm{d}$ & $\begin{array}{c}\mathrm{EMQ} \\
\%\end{array}$ & $\begin{array}{c}\mathrm{EMA} \\
\mathrm{W} \cdot \mathrm{m}^{-2}\end{array}$ & $\begin{array}{c}\text { ERM } \\
\%\end{array}$ & $\mathrm{c}$ & Interpretação & Categoria \\
\hline Bárbaro et al. (2010) & 2010 & 0,9906 & 0,9953 & 0,9976 & 0,8742 & 2,2269 & 0,0079 & 0,9929 & ÓTIMO & 3 \\
\hline Jin et al. (2006) & 2006 & 0,9898 & 0,9949 & 0,9975 & 0,9063 & 2,4160 & 0,0095 & 0,9924 & ÓTIMO & 3 \\
\hline Dilley \& O'Brien (1998) & 1998 & 0,9890 & 0,9945 & 0,9973 & 0,9428 & 2,3886 & 0,0062 & 0,9918 & ÓTIMO & 6 \\
\hline Satterlund (1979) & 1979 & 0,9767 & 0,9883 & 0,9946 & 1,2707 & 3,2806 & 0,1247 & 0,9830 & ÓTIMO & 3 \\
\hline Efimova (1961) & 1961 & 0,9726 & 0,9862 & 0,9938 & 1,3464 & 3,5594 & 0,1566 & 0,9801 & ÓTIMO & 3 \\
\hline Keding (1989) & 1989 & 0,9726 & 0,9862 & 0,9938 & 1,3464 & 3,5591 & 0,1562 & 0,9801 & ÓTIMO & 3 \\
\hline Angström (1918) & 1918 & 0,9726 & 0,9862 & 0,9938 & 1,3464 & 3,5590 & 0,1561 & 0,9801 & ÓTIMO & 3 \\
\hline Niemelä et al. (2001) & 2001 & 0,9726 & 0,9862 & 0,9938 & 1,3464 & 3,5590 & 0,1561 & 0,9801 & ÓTIMO & 3 \\
\hline $\begin{array}{l}\text { Zapadka et al (2001) - } \\
\text { modelo 2 }\end{array}$ & 2001 & 0,9726 & 0,9862 & 0,9938 & 1,3464 & 3,5590 & 0,1561 & 0,9801 & ÓTIMO & 3 \\
\hline $\begin{array}{l}\text { Berdhal \& Fromberg } \\
\text { (1982) mod2 }\end{array}$ & 1982 & 0,9725 & 0,9862 & 0,9938 & 1,3467 & 3,5609 & 0,1571 & 0,9801 & ÓTIMO & 4 \\
\hline Asklef (1928) & 1928 & 0,9723 & 0,9861 & 0,9938 & 1,3505 & 3,5805 & 0,1584 & 0,9799 & ÓTIMO & 3 \\
\hline Berdhal \& Martin (1984) & 1984 & 0,9723 & 0,9861 & 0,9938 & 1,3505 & 3,5805 & 0,1584 & 0,9799 & ÓTIMO & 3 \\
\hline Berger et al. (1984) mod1 & 1984 & 0,9723 & 0,9861 & 0,9938 & 1,3505 & 3,5805 & 0,1584 & 0,9799 & ÓTIMO & 3 \\
\hline Berland \& Berland (1952) & 1952 & 0,9723 & 0,9861 & 0,9938 & 1,3505 & 3,5805 & 0,1584 & 0,9799 & ÓTIMO & 3 \\
\hline Boutario (1932) & 1932 & 0,9723 & 0,9861 & 0,9938 & 1,3505 & 3,5805 & 0,1584 & 0,9799 & ÓTIMO & 3 \\
\hline Brunt (1932) & 1932 & 0,9723 & 0,9861 & 0,9938 & 1,3505 & 3,5805 & 0,1584 & 0,9799 & ÓTIMO & 3 \\
\hline
\end{tabular}

continua... 
Tabela 7: continuação...

\begin{tabular}{|c|c|c|c|c|c|c|c|c|c|c|}
\hline Modelo & Ano & $\mathrm{R}^{2}$ & $\mathrm{r}$ & $\mathrm{d}$ & $\begin{array}{c}\text { EMQ } \\
\%\end{array}$ & $\begin{array}{l}\text { EMA } \\
\text { W.m- }\end{array}$ & $\begin{array}{c}\text { ERM } \\
\%\end{array}$ & $\mathrm{c}$ & Interpretação & Categoria \\
\hline Chumanova (1947) & 1947 & 0,9723 & 0,9861 & 0,9938 & 1,3505 & 3,5805 & 0,1584 & 0,9799 & о́тIмо & 3 \\
\hline $\begin{array}{l}\text { De Coster \& Shuepp } \\
\text { (1957) }\end{array}$ & 1957 & 0,9723 & 0,9861 & 0,9938 & 1,3505 & 3,5805 & 0,1584 & 0,9799 & ÓTIMO & 3 \\
\hline Dines \& Dines (1927) & 1927 & 0,9723 & 0,9861 & 0,9938 & 1,3505 & 3,5805 & 0,1584 & 0,9799 & ÓTIMO & 3 \\
\hline FAO (1990) & 1990 & 0,9723 & 0,9861 & 0,9938 & 1,3505 & 3,5805 & 0,1584 & 0,9799 & ÓTIMO & 3 \\
\hline Gross \& Brooks (1957) & 1957 & 0,9723 & 0,9861 & 0,9938 & 1,3505 & 3,5805 & 0,1584 & 0,9799 & о́тімо & 3 \\
\hline Harshunova (1961) mod1 & 1961 & 0,9723 & 0,9861 & 0,9938 & 1,3505 & 3,5805 & 0,1584 & 0,9799 & о́тімо & 3 \\
\hline Heitor et al. (1991) & 1991 & 0,9723 & 0,9861 & 0,9938 & 1,3505 & 3,5805 & 0,1584 & 0,9799 & о́тIмо & 3 \\
\hline Iziomon et al (2003) mod 1 & 2003 & 0,9723 & 0,9861 & 0,9938 & 1,3505 & 3,5805 & 0,1584 & 0,9799 & ÓTIMO & 3 \\
\hline Korsgaard et al. (1991) & 1991 & 0,9723 & 0,9861 & 0,9938 & 1,3505 & 3,5805 & 0,1584 & 0,9799 & ÓTIMO & 3 \\
\hline $\begin{array}{l}\text { Lutherstein \& Chednovsky } \\
\text { (1946) }\end{array}$ & 1946 & 0,9723 & 0,9861 & 0,9938 & 1,3505 & 3,5805 & 0,1584 & 0,9799 & ÓTIMO & 3 \\
\hline Marshunova (1966) & 1966 & 0,9723 & 0,9861 & 0,9938 & 1,3505 & 3,5805 & 0,1584 & 0,9799 & ÓTIMO & 3 \\
\hline Monteith (1961) & 1961 & 0,9723 & 0,9861 & 0,9938 & 1,3505 & 3,5805 & 0,1584 & 0,9799 & ÓTIMO & 3 \\
\hline Robitsch (1926) & 1926 & 0,9723 & 0,9861 & 0,9938 & 1,3505 & 3,5805 & 0,1584 & 0,9799 & ÓTIMO & 3 \\
\hline Sellers (1965) & 1965 & 0,9723 & 0,9861 & 0,9938 & 1,3505 & 3,5805 & 0,1584 & 0,9799 & ÓTIMO & 3 \\
\hline $\begin{array}{l}\text { Swinbank (1963) - } \\
\text { modelo } 1\end{array}$ & 1963 & 0,9723 & 0,9861 & 0,9938 & 1,3505 & 3,5805 & 0,1584 & 0,9799 & о́тімо & 3 \\
\hline Viswanadham \& R. (1970) & 1970 & 0,9723 & 0,9861 & 0,9938 & 1,3505 & 3,5805 & 0,1584 & 0,9799 & о́тімо & 3 \\
\hline $\begin{array}{l}\text { Berdhal \& Fromberg } \\
\text { (1982) mod1 }\end{array}$ & 1982 & 0,9717 & 0,9857 & 0,9937 & 1,3620 & 3,6486 & 0,1600 & 0,9795 & ÓTIMO & 4 \\
\hline Berger et al. (1984) $\bmod 2$ & 1984 & 0,9717 & 0,9857 & 0,9937 & 1,3620 & 3,6486 & 0,1600 & 0,9795 & ÓTIMO & 4 \\
\hline Clark \& Allen (1978) & 1978 & 0,9716 & 0,9857 & 0,9936 & 1,3643 & 3,6609 & 0,1602 & 0,9794 & о́тімо & 4 \\
\hline Elsasser (1942) & 1942 & 0,9715 & 0,9856 & 0,9936 & 1,3674 & 3,6769 & 0,1606 & 0,9794 & ÓTIMO & 3 \\
\hline Greuell et al. (1997) & 1997 & 0,9652 & 0,9824 & 0,9924 & 1,4801 & 4,0587 & 0,1886 & 0,9750 & ÓTIMO & 3 \\
\hline Konzelmann et al (1994) & 1994 & 0,9652 & 0,9824 & 0,9924 & 1,4801 & 4,0587 & 0,1886 & 0,9750 & о́тIмо & 3 \\
\hline Sridhar et al. (2002) & 2002 & 0,9652 & 0,9824 & 0,9924 & 1,4801 & 4,0587 & 0,1886 & 0,9750 & о́тімо & 3 \\
\hline Kondo et al. (1990) & 1990 & 0,9651 & 0,9824 & 0,9924 & 1,4813 & 4,0633 & 0,1891 & 0,9749 & ÓTIMO & 6 \\
\hline Prata (1996) & 1996 & 0,9644 & 0,9820 & 0,9922 & 1,4908 & 4,1272 & 0,1907 & 0,9744 & о́TIMO & 6 \\
\hline $\begin{array}{l}\text { Iziomon et al. (2003) } \\
\text { mod 2e3 }\end{array}$ & 2003 & 0,9643 & 0,9820 & 0,9922 & 1,4928 & 4,1398 & 0,1919 & 0,9743 & о́тімо & 3 \\
\hline Klok \& Oerlemans (2002) & 2002 & 0,9639 & 0,9818 & 0,9921 & 1,4999 & 4,1748 & 0,1942 & 0,9741 & о́тімо & 3 \\
\hline Brutsaert (1975) & 1975 & 0,9636 & 0,9816 & 0,9921 & 1,5055 & 4,2041 & 0,1950 & 0,9738 & ÓTIMo & 3 \\
\hline Kruk et al. (2009) & 2009 & 0,9636 & 0,9816 & 0,9921 & 1,5055 & 4,2041 & 0,1950 & 0,9738 & ÓTIMO & 3 \\
\hline Lhomme et al. (2007) & 2007 & 0,9636 & 0,9816 & 0,9921 & 1,5055 & 4,2041 & 0,1950 & 0,9738 & о́тімо & 3 \\
\hline $\begin{array}{l}\text { Golaka \& Excell (2004) } \\
\text { mod1 }\end{array}$ & 2004 & 0,9414 & 0,9703 & 0,9856 & 2,1286 & 6,1480 & 0,0467 & 0,9562 & ÓTIMO & 1 \\
\hline $\begin{array}{l}\text { Swinbank (1963) - modelo } \\
2\end{array}$ & 1963 & 0,9379 & 0,9685 & 0,9847 & 2,1871 & 6,4618 & 0,0518 & 0,9537 & о́тімо & 1 \\
\hline Ohmura (1981) & 1981 & 0,9367 & 0,9678 & 0,9845 & 2,2009 & 6,5142 & 0,0581 & 0,9528 & ÓTIMO & 1 \\
\hline Deacon (1970) & 1970 & 0,9363 & 0,9676 & 0,9844 & 2,2053 & 6,5314 & 0,0592 & 0,9525 & о́тімо & 5 \\
\hline Idso \& Jackson (1969) & 1969 & 0,9340 & 0,9664 & 0,9839 & 2,2323 & 6,6263 & 0,0701 & 0,9509 & ÓTIMO & 1 \\
\hline Idso (1981) & 1981 & 0,9258 & 0,9622 & 0,9851 & 1,9896 & 5,7794 & 0,2864 & 0,9479 & ÓTIMO & 3 \\
\hline $\begin{array}{l}\text { Swinbank (1963) - modelo } \\
3\end{array}$ & 1963 & 0,9042 & 0,9509 & 0,9695 & 3,5676 & 11,6018 & 0,5100 & 0,9219 & ÓTIMO & 1 \\
\hline
\end{tabular}


Tabela 8: Avaliação dos modelos parametrizados para a estimativa da irradiância de ondas longas no cerrado Mato-grossense nas condições de céu parcialmente nublado $\mathrm{L}_{\mathrm{cld}}{ }^{*}$

\begin{tabular}{|c|c|c|c|c|c|c|c|c|c|c|}
\hline Modelo & $\overline{\text { Ano }}$ & $\mathrm{R}^{2}$ & $\bar{r}$ & $\bar{d}$ & $\begin{array}{l}\text { EMQ } \\
\%\end{array}$ & $\begin{array}{l}\text { EMA } \\
\text { W.m-2 }\end{array}$ & $\begin{array}{l}\text { ERM } \\
\%\end{array}$ & $\mathrm{c}$ & $\begin{array}{l}\text { Interpretação } \\
\text { Camargo \& } \\
\text { Sentelhas } \\
(1997)\end{array}$ & Categoria \\
\hline Aubinet (1994) - $\bmod 04$ & 1994 & 0,9446 & 0,9719 & 0,9881 & 1,7688 & 5,4495 & 0,2085 & 0,9603 & ÓTIMO & 3 \\
\hline $\begin{array}{l}\text { Bilbao \& de Miguel } \\
(2007) \bmod 2\end{array}$ & 2007 & 0,9438 & 0,9715 & 0,9880 & 1,7741 & 5,4896 & 0,2136 & 0,9598 & ÓTIMO & 3 \\
\hline Zapadka et al. (2007) & 2007 & 0,9436 & 0,9714 & 0,9879 & 1,7795 & 5,4451 & 0,2128 & 0,9597 & ÓTIMO & 3 \\
\hline Bignami et al. (1995) & 1995 & 0,9430 & 0,9711 & 0,9878 & 1,7830 & 5,4964 & 0,2162 & 0,9593 & ÓTIMO & 3 \\
\hline Aubinet (1994) - $\bmod 01$ & 1994 & 0,9412 & 0,9702 & 0,9853 & 2,0849 & 6,8166 & 0,0280 & 0,9559 & ÓTIMO & 1 \\
\hline $\begin{array}{l}\text { Parkinson \& Washington } \\
\text { (1979) }\end{array}$ & 1979 & 0,9367 & 0,9678 & 0,9845 & 2,1297 & 6,9866 & 0,0664 & 0,9528 & ÓTIMO & 1 \\
\hline Idso \& Jackson (1969) & 1969 & 0,9367 & 0,9678 & 0,9845 & 2,1301 & 6,9889 & 0,0663 & 0,9528 & ÓTIMO & 1 \\
\hline Lhomme et al. (2007) & 2007 & 0,9322 & 0,9655 & 0,9859 & 1,9002 & 6,0287 & 0,2428 & 0,9519 & ÓTIMO & 3 \\
\hline $\begin{array}{l}\text { Bilbao \& de Miguel } \\
(2007) \bmod 3\end{array}$ & 2007 & 0,9332 & 0,9660 & 0,9809 & 2,5518 & 8,3132 & 0,2114 & 0,9476 & ÓTIMO & 1 \\
\hline $\begin{array}{l}\text { Crawford and Duchon } \\
\text { (1999) }\end{array}$ & 1999 & 0,8866 & 0,9416 & 0,9747 & 2,5903 & 7,8986 & 0,4142 & 0,9177 & ÓTIMO & 7 \\
\hline Staley and Jurica (1972) & 1972 & 0,8811 & 0,9387 & 0,9748 & 2,5302 & 8,1192 & 0,4569 & 0,9150 & ÓTIMO & 8 \\
\hline
\end{tabular}

Tabela 9: Avaliação dos modelos parametrizados para a estimativa da irradiância de ondas longas no cerrado Mato-grossense nas condições de céu nublado $\mathrm{L}_{\text {cld }}{ }^{*}$

\begin{tabular}{|c|c|c|c|c|c|c|c|c|c|c|}
\hline Modelo & Ano & $\mathrm{R}^{2}$ & $\mathrm{r}$ & $\mathrm{d}$ & $\begin{array}{l}\text { EMQ } \\
\%\end{array}$ & $\begin{array}{l}\text { EMA } \\
\text { W.m-2 }\end{array}$ & $\begin{array}{c}\text { ERM } \\
\%\end{array}$ & c & Interpretação & Categoria \\
\hline Andreas \& Ackley (1982) & 1982 & 0,9957 & 0,9978 & 0,9989 & 0,5074 & 1,2462 & 0,0106 & 0,9968 & ÓTIMO & 3 \\
\hline Bignami et al. (1995) & 1995 & 0,9904 & 0,9952 & 0,9977 & 0,7267 & 1,8422 & 0,0597 & 0,9929 & ÓTIMO & 3 \\
\hline Zapadka et al. (2007) & 2007 & 0,9904 & 0,9952 & 0,9977 & 0,7267 & 1,8422 & 0,0597 & 0,9929 & ÓTIMO & 3 \\
\hline Lhomme et al. (2007) & 2007 & 0,9878 & 0,9939 & 0,9971 & 0,8096 & 2,0831 & 0,0729 & 0,9910 & ÓTIMO & 3 \\
\hline Idso \& Jackson (1969) & 1969 & 0,9668 & 0,9833 & 0,9917 & 1,4119 & 4,0220 & 0,0212 & 0,9751 & ÓTIMO & 1 \\
\hline $\begin{array}{l}\text { Crawford and Duchon } \\
\text { (1999) }\end{array}$ & 1999 & 0,8083 & 0,8991 & 0,9551 & 3,1018 & 7,3021 & 0,6589 & 0,8587 & ÓTIMO & 7 \\
\hline
\end{tabular}


Tabela 10: Comparação dos resultados estatísticos dos modelos originais com os modelos parametrizados na estimativa da irradiância de ondas longas no Cerrado Mato-grossense nas condições de céu claro, céu parcialmente nublado e céu nublado.

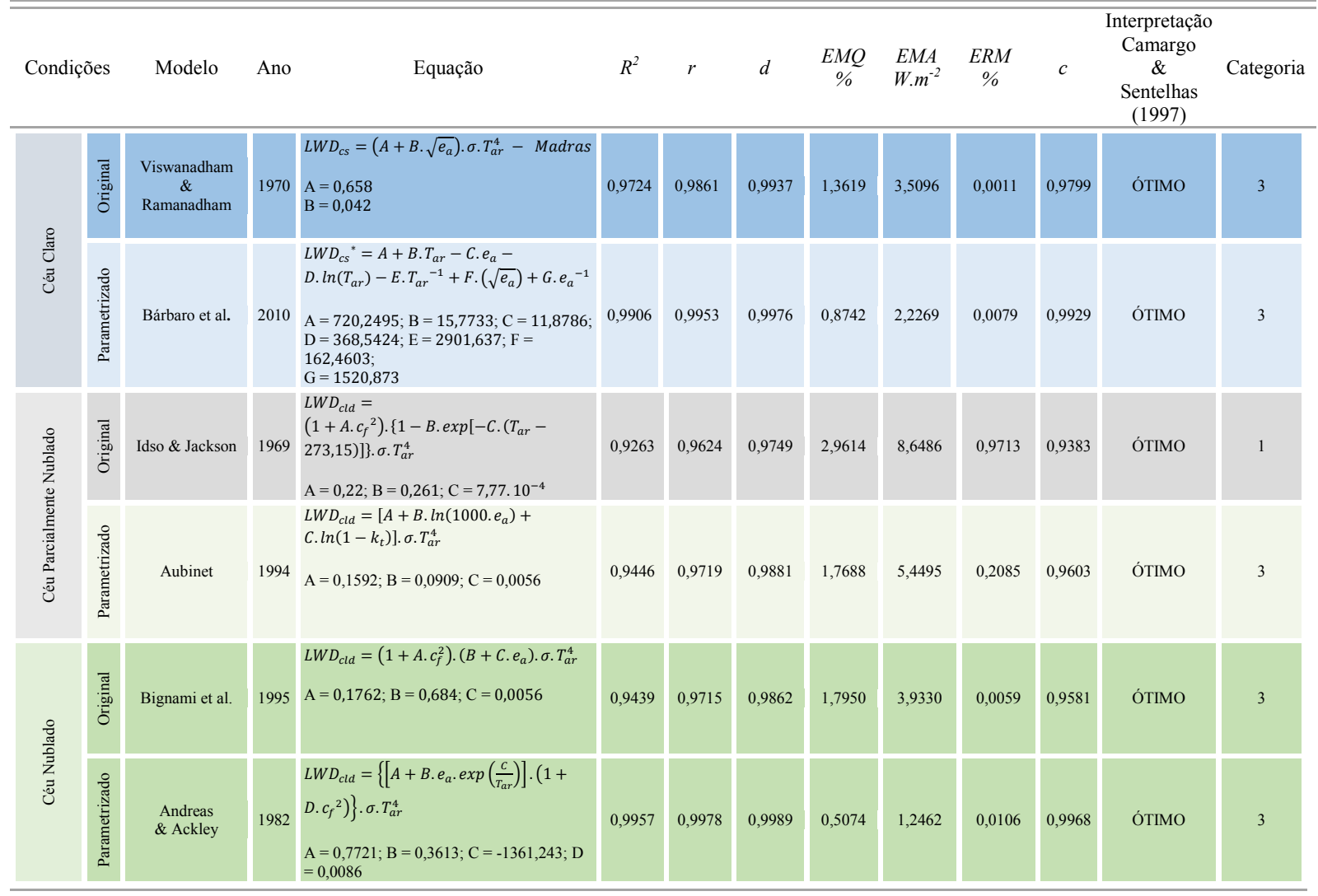

A tabela 10 mostra o resultado final após a avaliação dos 107 modelos e suas respectivas estatísticas $c, d$, re $R^{2}$ e em termos de ERM, EMA e EMQ.e a comparação destes resultados dos modelos originais de estimativa da irradiância de ondas longas para céu claro, parcialmente nublado e nublado que obtiveram o melhor desempenho nas condições do cerrado Mato-grossense com os modelos de melhor desempenho obtidos após a parametrização.

\section{CONCLUSÃO}

Dos cento e sete modelos analisados e parametrizados para as condições de céu claro, parcialmente nublado e nublado nas condições climatológicas do Cerrado Mato-grossense, seis modelos apresentaram os menores erros estatísticos e os maiores coeficientes como os de determinação $\left(R^{2}\right)$, de correlação de Pearson $(r)$ indicando uma correlação muito forte entre os valores estimados e os medidos, os melhores índices de concordância e, consequentemente, os melhores índices de desempenho.

Mesmo com seus parâmetros originais pode-se observar que os resultados obtidos não foram comprometidos visto que a maioria dos modelos teve um ótimo desempenho.

A maioria dos modelos propostos para as condições de céu claro $(0,65 \leq \mathrm{kT} \leq 1)$ apresentaram em sua formulação os parâmetros "pressão de vapor d'água no ar próximo a superfície" (ea) e a "temperatura do ar próximo à superfície" (Tar) e, para os modelos em que a presença de nuvens era considerado apresentou resultados satisfatórios visto que os modelos que a contemplava obtiveram alta correlação e desempenho em relação aos valores de $L d$ experimentais.

Nesta análise, podemos observar que os modelos de estimativa da irradiância de ondas longas, em sua formulação original, que apresentaram os melhores índices estatísticos e, portanto, aqueles que melhor se adaptaram ao Cerrado Mato-grossense, para o período estudado e para as condições de céu claro, parcialmente nublado e nublado foram, respectivamente, os modelos de Viswanadham \& Ramanadham (1970), Idso \& Jackson (1969) e Bignami et al. (1995) e, após a parametrização, os modelos de Bárbaro et al. (2010) (céu claro); Aubinet (1994) (céu parcialmente nublado) e Andreas \& Ackley (1982) (céu nublado). 


\section{REFERÊNCIAS BIBLIOGRÁFICAS}

AGUIAR, L. J. G. Balanço de radiação em áreas de floresta e de pastagem em Rondônia. 2007. 70f, Dissertação (Mestrado em Meteorologia Agricola) Universidade de Viçosa, Viçosa, 2007.

BERDAHL, P., MARTIN, M. Emissivity of clear skies. Solar Energy, v. 32, p. 663-664, 1984.

CAMARGO, A. P., SENTELHAS, P. C. Avaliação do desempenho de diferentes métodos de estimativa da evapotranspiração potencial no estado de São Paulo, Brasil. Revista Brasileira de Agrometeorologia, v.5, n.1, p.89-97, 1997.

BRUTSAERT, W. H. Evaporation Into the Atmosphere: Theory, history, and applications, Kluwer Acad. Norwell, Mass., 1982.

CAMARGO, A. P., SENTELHAS, P. C. Avaliação do desempenho de diferentes

métodos de estimativa da evapotranspiração potencial no estado de São Paulo, Brasil.

Revista Brasileira de Agrometeorologia, v.5, n.1, p.89-97, 1997.

DALLACORT, R.; FREITAS, P. S. L.; GONÇALVES, A. C. A.; REZENDE, R.; BERTONHA, A. Equações de estimativa da irradiação solar global, compartição mensal, para a região noroeste do Estado do Paraná. In: CONGRESSO NACIONAL DE IRRIGAÇÃO E DRENAGEM, 14., 2004, Porto Alegre, RS. 1 CD-ROM. Anais... Porto Alegre: ABID, 2004.

DUAN, Q., SCHAAKE, J., ANDR'EASSIAN, V., FRANKS, S., GOTETI, G., GUPTA, H. V., GUSEV, Y. M., HABETS, F., HALL, A., HAY, L., HOGUE, T., HUANG, M., LEAVESLEY, G., LIANG, X., NASONOVA, O. N., NOILHAN, J., OUDIN, L., SOROOSHIAN, S., WAGENER, T., WOOD, E. E. F. Model parameter estimation experiment (mopex): An overview of science strategy and major results from the second and third workshops, Journal of Hydrology, v. 320, p. 3-17.

EMBRAPA. Sistema brasileiro de classificação de solos. Brasília: Embrapa, p. 412, 1999.

FEITOSA, J. R. P., FERREIRA DA COSTA, R., FISCH, G.; SOUZA, S. S., NOBRE, C. A. Radiação solar global em áreas de floresta e pastagem na Ama- zônia. Aceito para publicação na Revista Brasileira de Agrometeorologia, 1998.

FOX, D. G. Judging air quality model performance, Bulletin of American Meteorological Society, v. 62, p. 599-609, 1980.

INMET, Observações meteorológicas, Instituto Nacional de Meteorologia, Cuiabá, INMET 1999.

LEGATES, D. R., MCCABE JR, E. G. J. Evaluating the use of "goodness-offit" measures in hydrologic and hydroclimatic model validation, Water Resources Research, v. 35 (1), p. 233-241. 1999.

MANTOVANI, J.E., PEREIRA, A. Estimativa da integridade da cobertura vegetal do Cerrado através de dados TM/Landsat. In: SIMPÓSIO BRASILEIRO DE SENSORIAMENTO REMOTO, Santos, SP. 9, 11-18/setembro/1998.

MANZI, A. O., VISWANADAM, Y., SÁ, L. D. A., ANDRÉ, R. G. B. Um estudo sobre o balanço de radiação da floresta Amazônica. São José dos Campos: INPE, 1986. (INPE-3956-PRE/974).

OKE, T. R. Boundary Layer Climates. London: Routledge, 1987. 435p.

STEWART, J. B.; THOM, A. S. Energy budgets in pine forest. Quarterly Journal of the Royal Meteorological Society, v. 99, n. 419, p. 154-170, 1973.

TANG, Y.; REED, P.; WAGENER, E. T. How effective and efficient are multiobjective evolutionary algorithms at hydrologic model calibration?, Hydrology and Earth System Sciences, v. 10, p. 289-307. 2006.

WILLMOTT C.J. Some comments on the evaluation of model performance. Bulletin of the American Meteorological Society, v. 63, p. 1309-1313, 1982.

WILLMOTT, C. J.; ACKLESON, S. G.; DAVIS, R. E.; FEDDEMA, H. J.; KLINK, K. M.; LEGATES, D.; O'DONNELL, R. J.; ROWE, E. C. M. Statistics for the evaluation and comparison of models. Journal of Geophysical Research, v. 90 (C5), p. 8995-9005. 1985.

YAPO, P. O.; GUPTA, H. V.; SOROOSHIAN, E S. Multi-objective global optimization for hydrologic models. Journal of Hydrology, v. 204, p. 83-97. 1998. 